\title{
Oxidative Kinetic Resolution of Aromatic Alcohols using Iron Nanoparticles
}

\author{
Jessica F. Sonnenberg, Dmitry Pichugin, Neil Coombs, \\ Robert H. Morris
}

\author{
Version Post-print/accepted manuscript \\ Citation Sonnenberg, J. F., Pichugin, D., Coombs, N., \& Morris, R. H. (2013). \\ (published version) Oxidative Kinetic Resolution of Aromatic Alcohols Using Iron \\ Nanoparticles. Topics in Catalysis, 56(13-14), 1199-1207.
}

Publisher's statement The final publication is available at Springer via

http:/ /dx.doi.org/10.1007/s11244-013-0086-2

How to cite TSpace items

Always cite the published version, so the author(s) will receive recognition through services that track citation counts, e.g. Scopus. If you need to cite the page number of the author manuscript from TSpace because you cannot access the published version, then cite the TSpace version in addition to the published version using the permanent URI (handle) found on the record page.

This article was made openly accessible by $U$ of $T$ Faculty. Please tell us how this access benefits you. Your story matters. 


\title{
Oxidative Kinetic Resolution of Aromatic Alcohols using Iron Nanoparticles
}

Jessica F. Sonnenberg, Dmitry Pichugin, Neil Coombs and Robert H. Morris*

Lash Miller Laboratories, Department of Chemistry, University of Toronto, 80 St. George Street, Toronto, Ontario, M5S 3H6

\begin{abstract}
Using our transfer hydrogenation (TH) pre-catalyst trans-(R,R)$\left[\mathrm{Fe}(\mathrm{NCMe}) \mathrm{CO}\left(\mathrm{PPh}_{2} \mathrm{C}_{6} \mathrm{H}_{4} \mathrm{CH}=\mathrm{NCHPh}-\right)_{2}\right]\left[\mathrm{BF}_{4}\right]_{2}$ (1) we investigated the reverse reaction, oxidative kinetic resolution (OKR), and were able to achieve turn-over frequencies up to $335 \mathrm{~h}^{-1}$ and s-values in favour of the (R)-alcohol up to 10.2. Using racemic 1-phenylethanol we optimized reaction conditions to maximize enantioselectivity and turn-over frequency (TOF) and studied the effect of different proton/hydride acceptors, temperatures, and bases. Using KOtBu as the base and benzophenone in $\mathrm{THF}$ as the solvent and acceptor at $45^{\circ} \mathrm{C}$, we tested a series of substrates with varying electronic and steric factors. By increasing the steric bulk at the alcohol, the enantioselectivity increased, however the TOF decreased dramatically. Varying the electronics of the substrates using electron withdrawing and donating substituents showed a less significant effect. We propose that the active species in catalysis is zero-valent iron nanoparticles (Fe NPs), a postulate that we support with microscopy imaging, sub-stoichiometric poisoning experiments, and analysis of the reaction profile. Further support is given in terms of a polymersupported substrate experiment whereby the active species in catalysis is too large to permeate the pores of a functionalized polymer. Our system is likely to be the first reported example of using a nanoparticle surface for oxidative kinetic resolution.
\end{abstract}

\section{Introduction}

The synthesis of enantiopure alcohols is of vital importance in the pharmaceutical, fragrance and food flavouring industries.[1] These alcohols are commonly made in industry via the selective 
hydrogenation of carbonyl groups by direct $\mathrm{H}_{2}$-hydrogenation or transfer hydrogenation (TH)[2] which uses isopropanol as the hydrogen source. Both types of hydrogenation typically employ homogeneous precious metal catalysts based on iridium, rhodium and ruthenium.[3] A newly expanding methodology for this type of transformation is the oxidative kinetic resolution of racemic alcohols to yield enantio-enriched alcohols and their corresponding ketones.[4] These reactions were first pioneered by Noyori[5] who used their homogeneous chiral diamine $\mathrm{Ru}^{\mathrm{II}} \mathrm{TH}$ catalyst as an oxidation catalyst for kinetic resolution. This is feasible because of the inherent reversibility of transfer hydrogenation catalysis, an approach to equilibrium process. For TH a proton and hydride are transferred from a donor, such as 2-propanol, to a ketone of interest, and is driven by the presence of an excess of 2-propanol. Therefore, in the presence of an appropriate acceptor, the reverse reaction is theoretically feasible with all TH catalysts.

An important current area of research is the use of 'greener' metal centres such as iron to replace toxic and expensive precious-metal catalysts.[6] Our group has developed several highly efficient transfer hydrogenation catalysts based on iron using PNNP tetradentate ligands.[7] We recently published that the active species in one of our catalytic systems is suspected to be zerovalent iron nanoparticles (Fe NPs) coated in chiral PNNP-ligand, with an overall diameter of 4.5 $\pm 1.2 \mathrm{~nm}$. [8] The precatalyst structure of trans-(R,R)-[Fe(NCMe)CO$\left(\mathrm{PPh}_{2} \mathrm{C}_{6} \mathrm{H}_{4} \mathrm{CH}=\mathrm{NCHPh}-\right.$ )$\left._{2}\right]\left[\mathrm{BF}_{4}\right]_{2}(\mathbf{1})$ and reaction scheme for this transformation and the reverse kinetic resolution oxidation reaction are depicted in Figure 1. An interesting aspect of this system was that it did not racemize enantiopure products during $\mathrm{TH}$ after prolonged exposure, which is often a problem with highly active systems both on iron[7b, 9] and on other precious-metal catalysts.[10] Given the reversibility of $\mathrm{TH}$ and this maintenance of enantiopurity over prolonged periods of time, we decided to test our suspected nanoparticle catalyst system for oxidative kinetic resolution of various substrates, and compare these results to known TH results for the same substrate series already developed.[7a] 
Figure 1: Precatalyst Structure and Reaction Schemes for Transfer Hydrogenation (TH) [Top] and Oxidative Kinetic Resolution [Bottom].

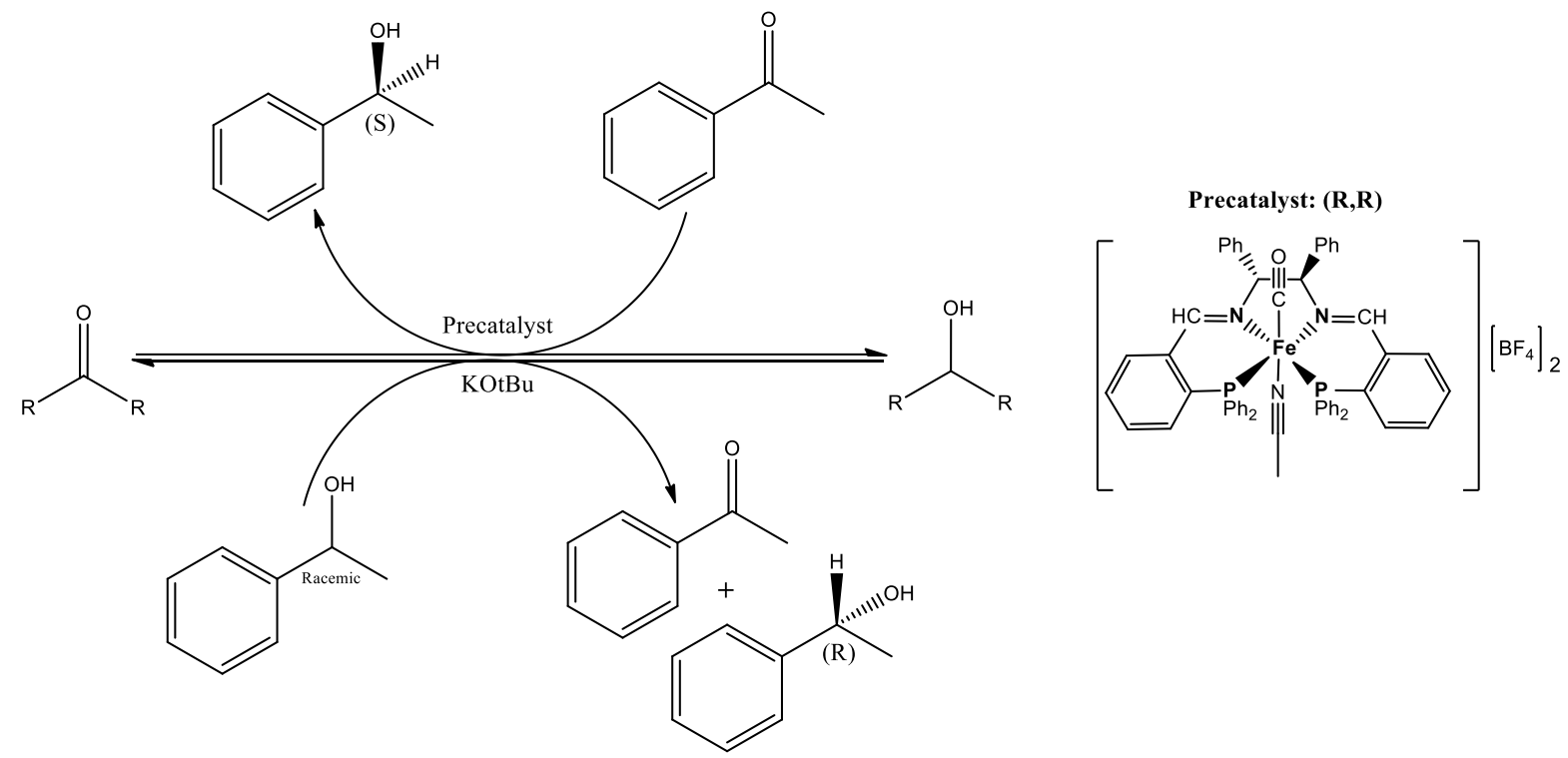

Different proton/hydride acceptors have also been explored, but the most common is acetone,[4b] and recent progress has been made using oxygen/air as the acceptor (aerobic oxidative kinetic resolution AOKR).[11] Several highly efficient catalytic systems have been developed previously for the kinetic resolution of alcohols using catalysts based on precious metals,[11-12] cobalt,[13] copper[14] and many more. However, the use of iron complexes for this transformation is rare,[15] and the use of nanoparticles or other heterogeneous catalysts have not yet been reported, to our knowledge.

\section{Results and Discussion}

\section{Catalytic Runs}

Acetophenone was typically used as the standard substrate to test $\mathrm{TH}$ with the iron catalysts in our lab, [7a, 8] so we first sought to explore the kinetic resolution and oxidation of racemic 1phenylethanol into acetophenone and the enantio-enriched alcohol. We have also reported that our active species for TH deactivates in the presence of oxygen,[8] and therefore we chose to use ketones as the proton/hydride acceptors instead of air. The results for tests with 1-phenylethanol are depicted in Table 1. Nanoparticle solutions for TH were typically formed by activating our 
iron precatalyst (1) with $\mathrm{KOtBu}$ in isopropanol at $28{ }^{\circ} \mathrm{C}$, then adding substrate. Therefore, paradoxically, base was employed to produce reactive alkoxides in order to reduce the iron centres and to allow for catalytic oxidations. Using acetone at $28{ }^{\circ} \mathrm{C}$ and $\mathrm{KOtBu}$ as the base yields only modest enantiomeric excess (e.e.) and a low relative rate of consumption of $\mathrm{R}$ versus $\mathrm{S}$ ('s' factor),[16] as shown in Entry 1. Selectivity for the (R)-alcohol is in keeping with TH results where the $(\mathrm{S})$-alcohol is preferentially produced. Enantiomeric excess in favour of (R)phenylethanol was significantly improved by using benzophenone in THF as the acceptor as shown in Entry 2. It should be noted that in changing the conditions from Entry 1 to 2, the concentration of acceptor also changes significantly; when acetone is used as the acceptor, it is also the solvent and is present at $82 \mathrm{mmol}$ (14000:1 relative to catalyst), whereas when benzophenone is used, it is present in much lower amounts of $7.7 \mathrm{mmol}$ (1000:1 relative to catalyst). The other very important reason for this change is the fact that acetone readily forms an enolate in the presence of strong base, but benzophenone does not as it has no enolizable protons. This would indicate that the formation of acetone enolates is potentially a deactivation pathway; either the base is consumed more rapidly by acetone and therefore is present in a lower concentration and cannot assist in the reduction of iron as readily, or the enolate competitively binds to the active species in the place of acetone, preventing the iron species from accepting the proton and hydride from the substrate to propagate the oxidation reaction.

The turn-over frequency (TOF) of reactions employing benzophenone as the acceptor were still low, so to improve the rate of the reaction, we increased the temperature from $28{ }^{\circ} \mathrm{C}$ to $45^{\circ} \mathrm{C}$ (Entry 3). We were pleased when the e.e. remained at 59\%, as did the value of 's', but the TOF increased significantly from 47 to $171 \mathrm{~h}^{-1}$. This indicates that the catalyst structure and mode of action in terms of selectivity is unchanged at higher temperatures, while greater reaction rates are obtained.

We were also interested in the effect of the base on the e.e., s-value and TOF of catalysis and therefore explored using the milder base sodium isopropoxide ( $\mathrm{NaOiPr}$ ) for catalysis. We hypothesized that using the strong base $\mathrm{KOtBu}$ in acetone was causing the formation of enolates, and therefore we wanted to test the use of a milder base with acetone, as depicted in entry 4 . The TOF increased significantly from 38 to 203, indicating that the same deactivation by enolates was not occurring. Although we were successful in increasing the reaction rate, the e.e. and s- 
value were significantly lower than the oxidation done using $\mathrm{KOtBu}$, indicating a severely diminished selectivity. To test whether this decrease in e.e. was an effect of changing the base, we also tested oxidation in benzophenone/THF at $45^{\circ} \mathrm{C}$ with $\mathrm{NaOiPr}$ (Entry 5). The TOF, e.e. and s-value were all slightly lower, indicating that the diminished e.e. and s-value in acetone were likely an effect of the base. The isopropoxide is more nucleophilic and may affect the selective binding of the substrate, thus diminishing the e.e.

Table 1: Kinetic Resolution of racemic 1-phenylethanol $(2.2 \mathrm{mmol})$ using $1(0.0056 \mathrm{mmol})$ and base $(0.045 \mathrm{mmol})$ [C:B:S = 1:8:400]

\begin{tabular}{|c|c|c|c|c|c|c|c|c|}
\hline Entry & $\begin{array}{l}\text { Temp } \\
\left({ }^{\circ} \mathrm{C}\right)\end{array}$ & Solvent & Base & $\begin{array}{l}\text { Time } \\
\text { (hours) }\end{array}$ & $\begin{array}{l}\text { Conversion } \\
(\% \mathrm{C}=\mathbf{O})\end{array}$ & $\begin{array}{l}\mathrm{TOF}^{\mathrm{c}} \\
\left(\mathrm{h}^{-1}\right)\end{array}$ & $\begin{array}{l}\text { e.e. }(\mathrm{R}) \\
(\%)\end{array}$ & $\begin{array}{l}\text { Relative } \\
\text { Rate (s) }\end{array}$ \\
\hline 1 & 28 & Acetone $^{b}$ & $\mathrm{KOtBu}$ & 5 & 43 & 38 & 40 & 4.6 \\
\hline 2 & 28 & $\mathrm{THF}^{\mathrm{a}}$ & $\mathrm{KOtBu}$ & 5 & 51 & 46 & $\begin{array}{l}59(5 \mathrm{~h}) \\
70(6 \mathrm{~h})\end{array}$ & 6.2 \\
\hline 3 & 45 & $\mathrm{THF}^{\mathrm{a}}$ & $\mathrm{KOtBu}$ & 1.5 & 52 & 171 & 59 & 6.0 \\
\hline 4 & 28 & Acetone $^{\mathrm{b}}$ & $\mathrm{NaOiPr}$ & 1 & 49 & 203 & $\begin{array}{l}33(1 \mathrm{~h}) \\
86(3 \mathrm{~h})\end{array}$ & 2.8 \\
\hline 5 & 45 & $\mathrm{THF}^{\mathrm{a}}$ & $\mathrm{NaOiPr}$ & 1.5 & 51 & 130 & 51 & 4.9 \\
\hline
\end{tabular}

${ }^{\mathrm{a}}$ Reaction done in stock solution of benzophenone $(1.4 \mathrm{~g}, 7.7 \mathrm{mmol})$ in THF $(4.7 \mathrm{~mL}, 58 \mathrm{mmol})$

${ }^{\mathrm{b}}$ Reaction done in acetone $(6 \mathrm{~mL}, 82 \mathrm{mmol})$

${ }^{\mathrm{c}}$ TOF calculated based on slope of linear portion of conversion plot relative to catalyst concentration

Having optimized the reaction conditions for our system using 1-phenylethanol as the substrate, we were interested in exploring sterically and electronically different substrates, as depicted in Table 2. We first examined the effect of increasing the sterics of the substrate, in an attempt to increase the e.e. as had been seen previously for TH.[7a] Starting with the most bulky substrate 2,2-dimethyl-1-phenylpropanol (Entry 2) there was no conversion to the corresponding ketone even at lower substrate loadings and prolonged reaction times. Decreasing the steric bulk slightly we tested 2-methyl-1-phenylpropanol (Entry 3) for oxidative kinetic resolution and were able to achieve an s-value of 10.2; however the reaction required lower substrate loadings, prolonged reaction times, and gave a very low TOF of $13 \mathrm{~h}^{-1}$. Completing the series of substrates, we tested 1-phenylpropanol (Entry 4) which is only slightly bulkier than 1-phenylethanol, and were able to achieve an s-value of 8.9 and a TOF of 123 . This series very clearly shows that with increasing steric bulk the e.e. (and therefore s-value) increases and the TOF (and therefore rate of reaction) decreases. This behaviour closely mimics that seen for TH with this system,[7a] supporting the truly reversible nature of these reactions. 
We were also interested in the effect of changing the electronics of the substrate on catalysis. We studied 1-phenylethanol substrates with electron withdrawing chloro- substituents in the para (Entry 5) and meta (Entry 6) positions. Both showed similar TOF of $\sim 90 \mathrm{~h}^{-1}$, lower than that observed for the unsubstituted 1-phenylethanol (TOF = 171), with s-values of 4.0-4.5, also lower than the unsubstituted substrate $(\mathrm{s}=6.0)$. A similar trend of decreasing rate and e.e. was observed for TH with the meta-substituted chloro substrate; however the para-substitution had a negligible effect on TH.[7a] To explore electron donating effects, we studied the para-methyl substituted substrate (Entry 7). The TOF was the highest of our series of substrates at $335 \mathrm{~h}^{-1}$, with an s-value of 5.3, comparable to 1-phenylethanol. Electron donation from the para position can be delocalized through the phenyl ring making the alcohol more reducing and therefore more rapidly oxidized, increasing the observed rate. The trend for the series of electron withdrawing through electron donating groups is less clear than with the steric modifications, but it is clear that the effect of changing the electronics is less significant than the effect of changing the sterics.

The last substrate of interest was 4-phenyl-2-butanol (Entry 8), which does not have the C-O bond in conjugation with the phenyl ring. Catalysis with prolonged reaction times and low substrate loading yielded very low TOF and poor selectivity, indicating that some steric bulk is required around the $\mathrm{C}-\mathrm{O}$ bond to allow for enantioselectivity, and that electronics are important for influencing reaction rates.

Table 2: Kinetic Resolution of various racemic alcohols using $1(0.0056 \mathrm{mmol})$ and KOtBu $(0.045 \mathrm{mmol})$ at $45^{\circ} \mathrm{C}$ in a stock solution of benzophenone $(1.4 \mathrm{~g}, 7.7 \mathrm{mmol})$ in THF $(4.7 \mathrm{~mL}, 58 \mathrm{mmol})$

\begin{tabular}{|l|l|l|l|l|l|l|l|}
\hline Entry & Product/Substrate & $\begin{array}{l}\text { Substrate } \\
\text { Loading }\end{array}$ & $\begin{array}{l}\text { Time } \\
(\mathbf{h})\end{array}$ & $\begin{array}{l}\text { Conversion } \\
\mathbf{\%} \mathbf{C}=\mathbf{O}\end{array}$ & $\begin{array}{l}\text { TOF } \\
\left(\mathbf{h}^{-1}\right)\end{array}$ & $\begin{array}{l}\text { e.e. (R) } \\
(\boldsymbol{\%})\end{array}$ & $\begin{array}{l}\text { Relative } \\
\text { Rate (s) }\end{array}$ \\
\hline 2 & 400 & 1.5 & 52 & 171 & 59 & N/A \\
\hline 3 & 110 & 12 & 0 & 0 & 0 & 10.2 \\
\hline
\end{tabular}




\begin{tabular}{|l|l|l|l|l|l|l|l|}
\hline 4 & 400 & 3 & 52 & 123 & 71 & 8.9 \\
\hline 6 & 400 & 2.5 & 50 & 91 & 48 & 4.0 \\
\hline 8 & 400 & 0.75 & 51 & 51 & 56 & 535 \\
\hline 5
\end{tabular}

\section{Evidence for Nanoparticles}

Previously we reported an in-depth series of experiments to examine the potential heterogeneity of the TH catalytic system using (1),[8] and we were interested in whether iron (0) nanoparticles (Fe NPs) were also the active species for the oxidative kinetic resolution detailed here. Scanning Transmission Electron Microscopy (STEM) is a powerful imaging tool for determining whether nanoparticles are present during catalysis.[17] STEM imaging for the present work was done at $100{ }^{\circ} \mathrm{C}$ using an Hitachi HD-2000 STEM on carbon/copper grids coated with activated catalytic solutions. Low temperatures were needed for analysis to minimize organic contamination by the electron beam.

The first experiment was done on a sample prepared by activating precatalyst (1) with NaOiPr in acetone, and using isopropanol as the substrate for 12 minutes with a C:B:S loading of 1:8:230. Isopropanol was used as the substrate as it has a much lower boiling point than 1-phenylethanol, and it could therefore be evaporated off the grid prior to analysis. Imaging showed reasonably well dispersed nanoparticles with an average diameter of 4-5 nm, as shown in Figure 2 [left]. These are similar in size and distribution to the nanoparticles previously reported,[8] as well as other $\mathrm{Fe}(0)$ NPs in the literature.[18] 
We were also interested in whether the other modes of activation involving other acceptor molecules would yield similarly dispersed nanoparticles. The second experiment was done on a sample prepared by activating precatalyst (1) with KOtBu in a benzophenone/THF solution, using isopropanol as the substrate for 20 minutes, with a $\mathrm{C}: \mathrm{B}: \mathrm{S}$ loading of 1:8:230. Imaging was not as clear as it was for solutions activated in acetone because the benzophenone cannot be removed from the grids under vacuum prior to imaging. This resulted in large organic masses being present during analysis as depicted in Figure 2 [right]. The images do still show that there are small nanoparticles on the grid behind the organics, and that they are also 4-5 $\mathrm{nm}$ in diameter. Another interesting feature of the images using benzophenone is the texture that can be observed encircling the organic masses; several of the benzophenone clouds appear to have bright rings around them, identified as being salts $\left(\mathrm{KBF}_{4}\right)$ which dried surrounding the organic masses.

Figure 2: STEM image taken at $-\mathbf{1 0 0}^{\circ} \mathrm{C}$ of activated catalyst; a) [left] solution prepared using (1) and $\mathrm{NaOiPr}$ at room temperature $\left(28^{\circ} \mathrm{C}\right)$ in acetone, using iPrOH as the substrate $(\mathrm{C}: \mathrm{B}: \mathrm{S}=1: 8: 230)$; b) [right] solution prepared using (1) and KOtBu at room temperature $\left(28^{\circ} \mathrm{C}\right)$ in benzophenone/THF, using $\mathrm{IPrOH}$ as the substrate $(\mathrm{C}: \mathrm{B}: \mathrm{S}=1: 8: 230)$.
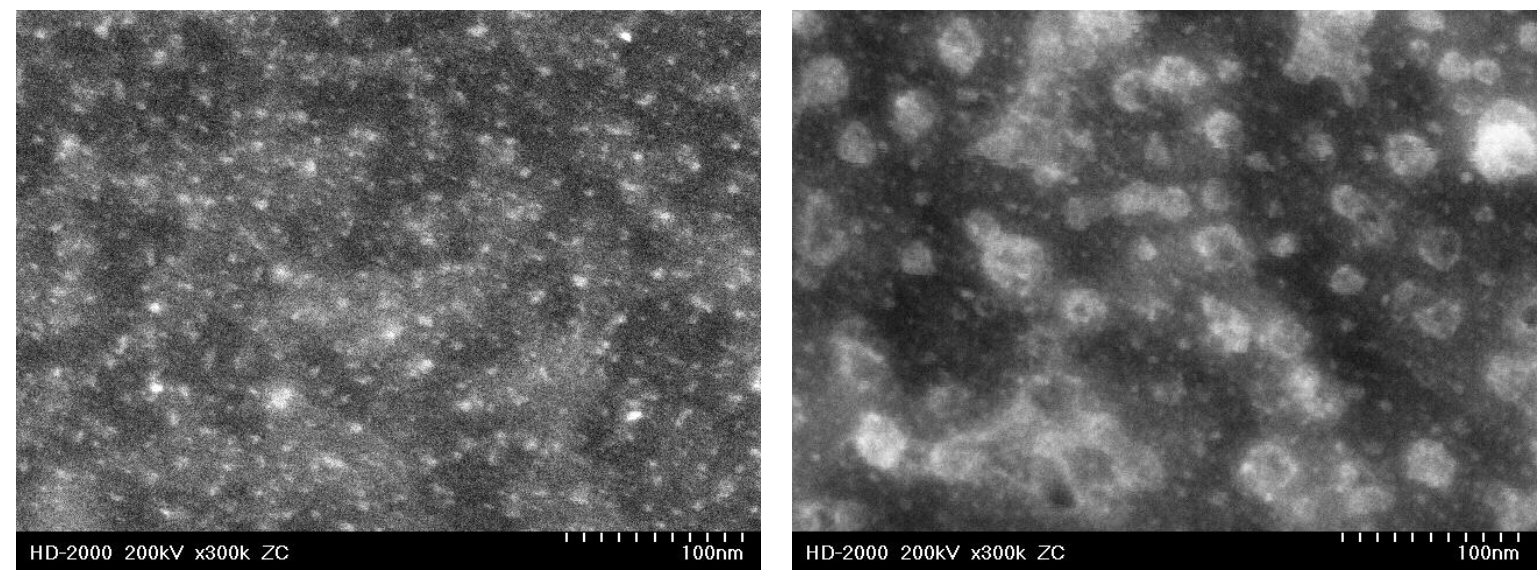

STEM, although a powerful tool for studying the structure of nanoparticles and determining whether they are present during catalysis, does not prove whether the nanoparticles are the active species in catalysis. For this, in situ experiments are needed to probe the activation and the active species.[8, 17] Similar to the analysis of the active species for $\mathrm{TH}$, we were interested in studying the reaction profile for clues as to the mode of activation of the iron precursor. Similar to what was observed for $\mathrm{TH}$, the reaction profile for oxidation of 1-phenylethanol at $45^{\circ} \mathrm{C}$ in benzophenone/THF is sigmoidally shaped, as depicted by the blue curve of Figure 3. This ' $\mathrm{S}$ ' shape of the reaction curve with an induction period, followed by rapid catalytic activity, then 
equilibration is indicative of colloid catalysis whereby the iron (II) complex first needs to be reduced and form the active nanoparticles.[17] Studying the induction period is a useful way of determining the mode of activation. Reactions were done where the precatalyst (1) was reacted with $\mathrm{KOtBu}$ in either 1 -phenylethanol at $45{ }^{\circ} \mathrm{C}$ for 10 minutes prior to $\mathrm{THF} /$ benzophenone addition [purple plot] or in THF/benzophenone at $45^{\circ} \mathrm{C}$ for 10 minutes prior to substrate addition [red plot]. In the case of pre-mixing with THF/benzophenone, no induction period is observed, as is shown in Figure 3 [red]. Subsequently, we tested the effect of pre-mixing the iron and KOtBu with 1-phenylethanol prior to addition of THF/benzophenone. No induction period is observed, and the reaction initiates much more rapidly as seen in Figure 3 [purple]. An alcohol solution containing base is going to be significantly more reducing than a THF/benzophenone solution containing base, supporting the results that the active species forms much more rapidly when it is pre-activated in alcohol than when it is pre-activated in solvent/acceptor, as would be expected for zero-valent nanoparticles. These results also show that the induction period is caused by the reaction of iron with base, and that the substrate alcohol does not form unwanted species like enolates when pre-mixed with the active species, as we have observed with other iron species in our group.[19] 
Figure 3: Reaction profiles for the catalytic oxidation of racemic 1-phenylethanol $(2.2 \mathrm{mmol})$ to acetophenone at $45^{\circ} \mathrm{C}$ in THF with benzophenone $(7.7 \mathrm{mmol})$, and runs in which the solvent (THF/benzophenone) or substrate (1-phenylethanol) were added at $45^{\circ} \mathrm{C} 10$ minutes prior to reaction commencement

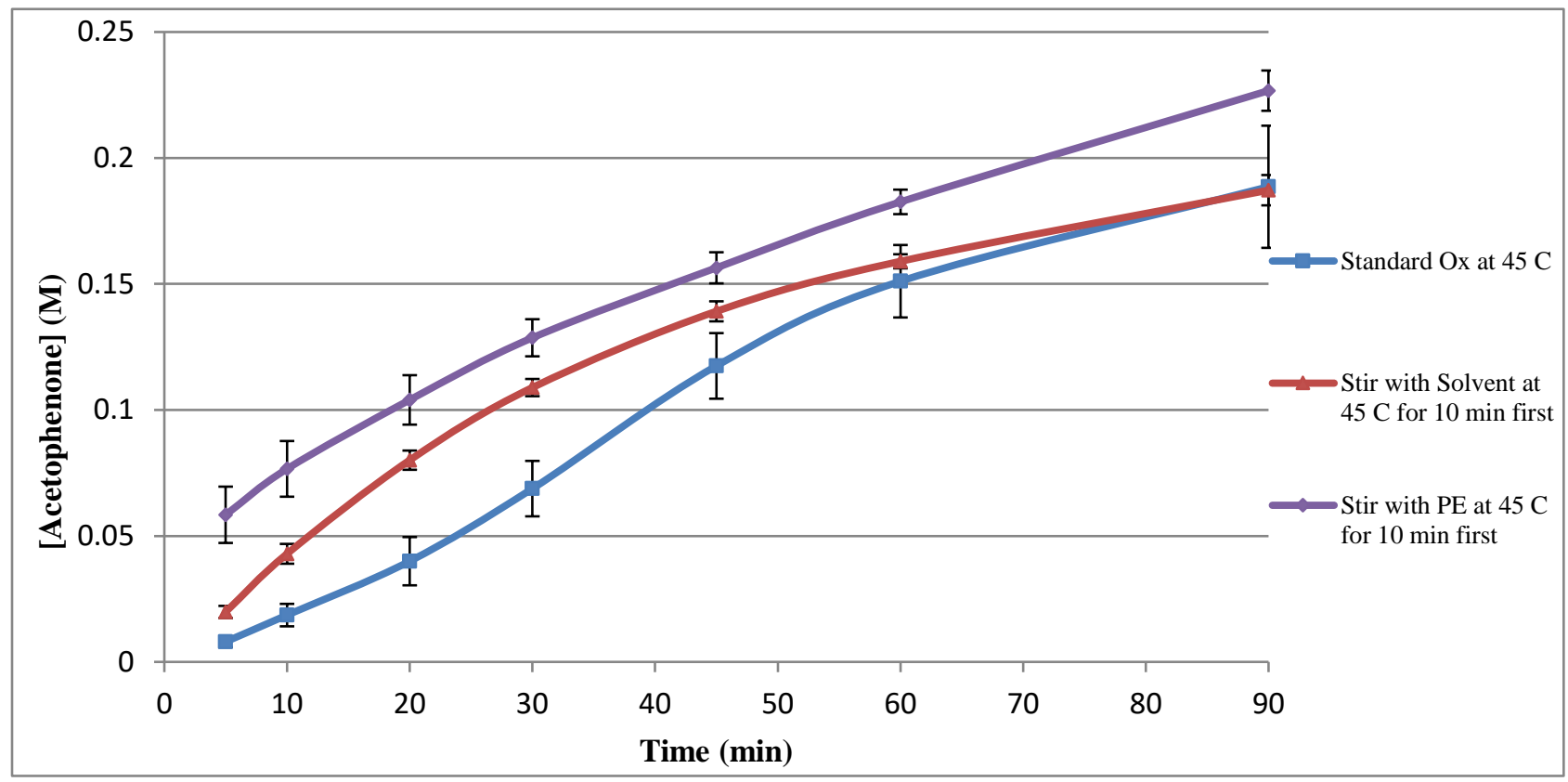

We were also interested in the effect this order of addition had on the resultant e.e. of the alcohols. The e.e. versus time plot that corresponds with Figure 3 is shown in Figure 4, and has several interesting features. The curve for the standard oxidation run [blue] shows a sigmoidal shape very similar to the conversion profile, and has a resultant e.e. of $62 \%$ at $90 \mathrm{~min}$ and $69 \%$ after $2 \mathrm{~h}$. The profile for pre-activating iron complex (1) and base in THF/benzophenone [red] once again shows no induction period, with a monatomic increase, but has the identical resultant e.e. This indicates that during the reaction of iron and base without substrate present, the same asymmetric active species is formed. The main difference in the two plots is that the active species is either allowed to preform and is therefore immediately active when substrate is added (red), or to slowly form in the presence of substrate yielding the induction period (blue). This also provides further support that uptake of substrate is not responsible for the induction period, but rather the reduction of the iron (II) complex to zero valent nanoparticles coated in chiral ligand. The last curve in Figure 4 [purple] shows the effect of pre-mixing the iron complex and base in 1-phenylethanol before the addition of THF/benzophenone. This once again yields no induction period; however the resultant e.e. is significantly lower, at only $36 \%$ after $90 \mathrm{~min}$, and $39 \%$ after $2 \mathrm{~h}$. Using KOtBu in 1-phenylethanol will yield much more reducing conditions, allowing for a more facile formation of zero-valent iron species, which would cause the 
increased activity in this case. This will also form a racemic mixture of phenyl-alkoxides, which, if the reaction proceeds via an inner-sphere type mechanism, could coat a nanoparticle surface before the THF/benzophenone is added. This increase in racemic alkoxide binding could result in less order, causing the decreased e.e. observed. A homogeneous explanation of this behaviour could be given in terms of an iron species (potentially a dimer or cluster compound) with multiple alkoxide binding sites.

Figure 4: Corresponding enantiopurity in (R)-phenylethanol over time profile to Figure 3.

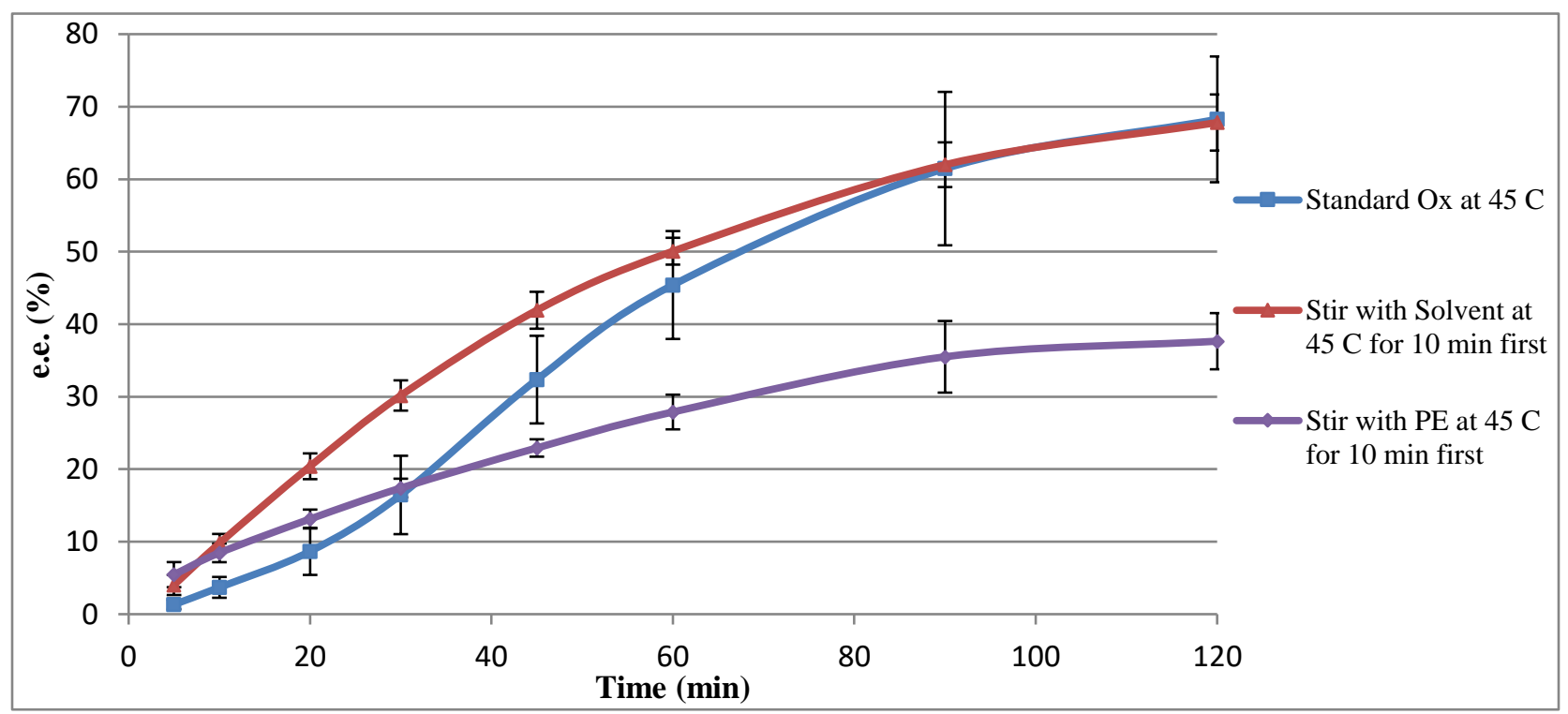

It is commonly reported that the use of sub-stoichiometric amounts of small phosphines and sulphides such as $\mathrm{PPh}_{3}$ and $\mathrm{CS}_{2}$ as poisoning agents for catalysis is evidence for the formation of NPs. $[17,20]$ We previously studied a large series of potential poisoning agents for $\mathrm{Fe}(0) \mathrm{NPs}$ and determined that trimethylphosphine $\left(\mathrm{PMe}_{3}\right)$ in toluene at only $10 \%$ loading relative to precatalyst could stop or significantly impede TH catalysis.[8] OKR experiments were run by initiating catalysis of 1-phenylethanol by standard methods, followed by addition of $15 \% \mathrm{PMe}_{3}$ relative to complex (1) after a set period of time. Poisoning was tested for catalysis with both types of acceptor molecules (acetone and benzophenone) under their optimized reaction conditions as depicted in Figure 5. Using (1), NaOiPr, acetone, 1-phenylethanol and poisoning with $\mathrm{PMe}_{3}$ at $\mathrm{t}=11 \mathrm{~min}$, the profile [purple] when compared to the non-poisoned run [green], shows a significant decrease in activity. The profile does not completely plateau, and therefore the active species is not fully poisoned, but this does show that with only $15 \% \mathrm{PMe}_{3}$ relative to 
Fe the majority of active sites have become bound and inactive, indicative of nanoparticle catalysis. Similarly, for the oxidation of 1-phenylethanol in benzophenone/THF using KOtBu as the base at $45{ }^{\circ} \mathrm{C}, 15 \% \mathrm{PMe}_{3}$ was added to the reaction mixture at $\mathrm{t}=21 \mathrm{~min}$. When comparing the poisoned profile [red] to the non-poisoned profile [blue] the same trend of a significant decrease, but not complete cessation in activity is observed, once again supporting Fe (0) NP catalysis.

Figure 5: Standard and poisoned catalytic runs for the oxidation of 1-phenylethanol in acetone using $\mathrm{NaOiPr}$ at $28^{\circ} \mathrm{C}$ and in benzophenone/THF using KOtBu at $45^{\circ} \mathrm{C}$, with $\mathrm{C}: \mathrm{B}: \mathrm{S}=1: 8: 400$

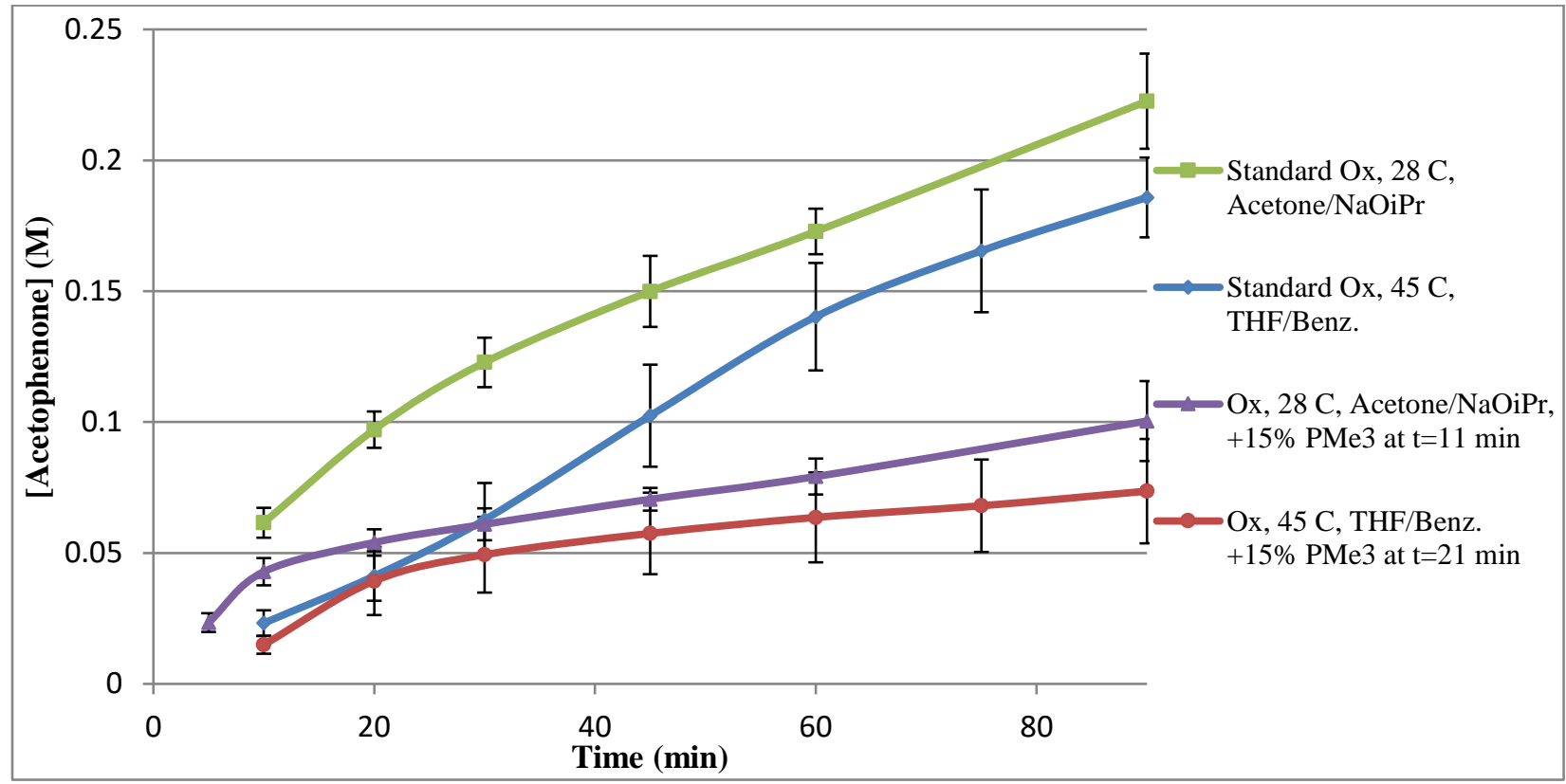

Following from the effect the poison had on the rate of the reaction, we were interested in the poison's effect on the e.e. as we have previously reported that poisons have no effect on the e.e. for TH.[8] To study this effect, we compared the e.e. of the alcohols to the concentration of acetophenone formed throughout the reaction for both non-poisoned and poisoned runs. To make an accurate comparison, the poisoned catalytic systems needed to be run for $\geq 6 \mathrm{~h}$ and only achieved an average of $32 \%$ conversion. Plotting the [acetophenone] (M) versus the e.e. (\%) [see Supporting Information] for poisoned and non-poisoned experiments for both types of solvent/acceptors (acetone and THF/benzophenone) yielded the same trend. The poisoned and non-poisoned profiles (for both sets of oxidation experiments) showed the same slope and shape of curve indicating that the e.e. is not affected by the addition of a poisoning agent. This is significant as it shows that the poisoning agents simply bind to the active sites, blocking 
substrate from binding, and do not alter the structure or functionality of the active site in the process. This type of behaviour would also be expected for nanoparticle catalysis.

Our last series of experiments to probe the potential heterogeneity of the active species involved tethering our standard substrate, 1-phenylethanol, to the interior of a porous polymer resin and investigating the catalytic behaviour. The goal of this experiment was to use the porous polymer as a type of 'size-exclusion sieve' to gauge the overall size of the active catalytic species. The experiment involved using organic reactions to tether a substrate that the active species can oxidize within a polymer, then compare catalysis using (1) with another small, well-known reagent (pyridinium chlorochromate-PCC) on the polymer, and analyze whether the substrate was oxidized. Small reagents such as PCC should be able to fit within the pores of the polymer and oxidize the phenylethanol to acetophenone, whereas nanoparticles would be too large to fit within the pores, resulting in no conversion to ketone. This concept has been used in the past by our lab[8] and by other research groups.[21] To analyze the polymers and to determine their functionality, the polymer beads were swollen using deuterated dichloromethane and protondecoupled carbon NMR was run on an Agilent DD2 $600 \mathrm{MHz}$ spectrometer using a $3.2 \mathrm{~mm}$ narrow bore HXY solid state probe. The polymers needed to be swollen prior to running NMR in order to get adequate signal strength and resolution of peaks.[22] The desired functionality was installed using the commercially available Wang resin with a $1.0-1.5 \mathrm{mmol}-\mathrm{OH} / \mathrm{g}$ polymer of benzyl alcohol functionality. This was converted to a benzaldehyde functionality (Wang-B) using pyridinium chlorochromate in dichloromethane, which could then be converted into the desired 1-phenylethanol functionality (Wang-PE) using an excess of methylmagnesium chloride in tetrahydrofuran, as depicted in Figure 6. 


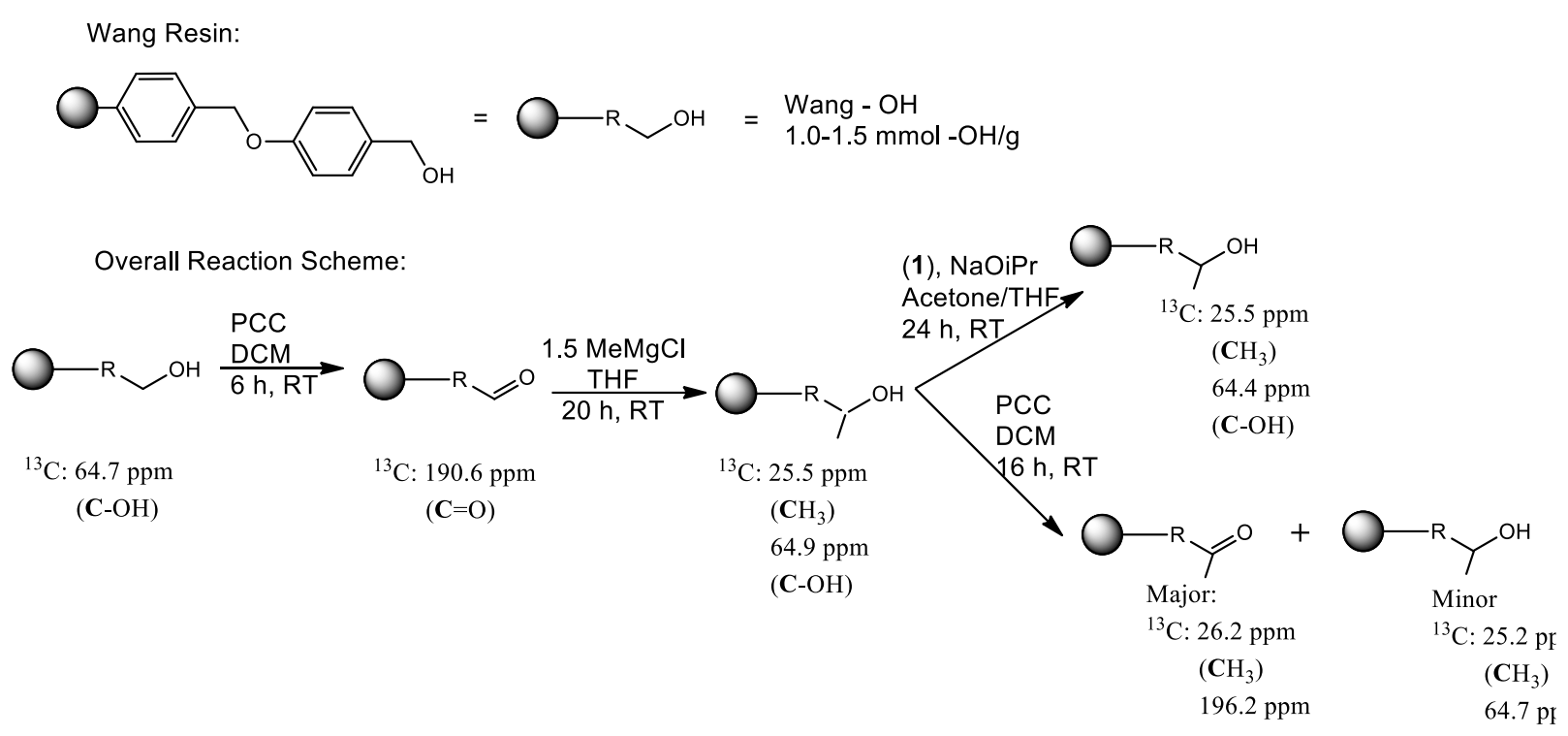

To probe whether known reagents could penetrate the pores of the polymer, Wang-PE was treated with PCC in dichloromethane for $16 \mathrm{~h}$. NMR showed the appearance of a ketone carbonyl carbon resonance at $196.2 \mathrm{ppm}$ and the corresponding $-\mathrm{CH}_{3}$ resonance at $26.2 \mathrm{ppm}$ as the major species, and Wang-PE's $\mathbf{C}-\mathrm{OH}$ and $-\mathrm{CH}_{3}$ peaks as the minor species, indicating that an acetophenone functionalized polymer had formed, proving that large metal complexes can permeate the polymer. The highest TOF we achieved using (1) for the oxidation of 1phenylethanol involved using acetone and $\mathrm{NaO} i \operatorname{Pr}$ at $28^{\circ} \mathrm{C}$, and hence were the conditions we used for Wang-PE. The reaction was done at a much higher substrate loading (1:35 catalyst to C$\mathrm{OH}$, versus 1:400 as typically employed), for $24 \mathrm{~h}$ instead of $2 \mathrm{~h}$, and in the presence of THF to maximize pore swelling and potential reactivity.[8, 23] NMR showed no change in ${ }^{13} \mathrm{C}$ signals from the original Wang-PE and no appearance of ketone signals as had been seen for oxidation with PCC, indicating that oxidation did not occur using (1). This implies that the active species during oxidative kinetic resolution is too large to permeate the pores of the polymer, further suggesting that the active species during catalysis are zero-valent nanoparticles. Similar to the $\mathrm{TH}$ case, it is never possible to conclusively prove that nanoparticles are the active species in catalysis. However we present substantial evidence that supports that $\mathrm{Fe}(0) \mathrm{NPs}$ are the active species in the oxidative kinetic resolution of aromatic alcohols using (1) as the pre-catalyst. 


\section{Conclusions}

Using our trans-[Fe( $\left.\mathrm{NCMe}) \mathrm{CO}\left(\mathrm{PPh}_{2} \mathrm{C}_{6} \mathrm{H}_{4} \mathrm{CH}=\mathrm{NCHPh}-\right)_{2}\right]\left[\mathrm{BF}_{4}\right]_{2}$ precatalyst, we studied the reversibility of asymmetric $\mathrm{TH}$ in terms of oxidative kinetic resolution of racemic aromatic alcohols. Initially studying 1-phenylethanol as our substrate, we probed different bases, sacrificial proton/hydride acceptors and temperatures and were able to achieve high TOF (> 200 $\mathrm{h}^{-1}$ ) using acetone and $\mathrm{NaOiPr}$ as well as reasonable e.e. and s-values (59\% and 6.0) at 50\% conversion to acetophenone using $\mathrm{THF} /$ benzophenone and $\mathrm{KOtBu}$ at $45^{\circ} \mathrm{C}(\mathrm{TOF}=171)$. Using these optimized conditions we tested a wide range of sterically and electronically varied substrates. By increasing the steric bulk at the alcohol, the rates of oxidation decreased dramatically, however the e.e. and s-value increased significantly. Varying the electronics of the substrate did not give such a linear relationship; by adding para-electron donating groups the rate increased significantly, and by adding electron withdrawing chloro groups at either the meta or para positions, the rate and selectivity decreased slightly. To investigate the true nature of the active species we analysed the reaction mixtures by STEM and were able to image small 4-5 nm nanoparticles, proving that they are formed during catalysis. To test whether nanoparticles are the active species, we were able to demonstrate that the reaction profile is sigmoidal in shape and that the induction period is caused by the reaction of iron with base, not the uptake of substrate. This supports the claim that the iron precursor must first be reduced to $\mathrm{Fe}(0)$ to form nanoparticles, which presumably are coated with chiral PNNP ligand, as we hypothesized for the reverse TH active species.[8] We also explored $\mathrm{PMe}_{3}$ as a sub-stoichiometric poisoning agent. At $15 \%$ loading of poison relative to iron, we were able to show that the catalysis is significantly diminished, further supporting that nanoparticles coated in chiral ligand are the active species for catalysis. Lastly, we probed the size of the active species using polymer-supported substrate experiments to show that the active species is too large to fit within the pores of a polymer resin. To the best of our knowledge, this is the first example of oxidative kinetic resolution occurring on the surface of a nanoparticle. It is also an interesting example of a reversible asymmetric reaction being shown to operate in both the forward and reverse direction on the surface of a nanoparticle. 


\section{Associated Content:}

Supporting Information. Experimental procedures, plots of all catalytic runs and poisoning experiments including error bars, STEM imaging and all solid state NMR plots are available online.

\section{Author Information:}

Corresponding Author. Correspondence should be addressed to R.H.M. at the Department of Chemistry, University of Toronto, 80 St George Street, Toronto, Ont. M5S3H6 rmorris@chem.utoronto.ca

Author Contributions. All authors have given approval to the final version of the manuscript.

\section{Acknowledgements:}

We thank the Natural Sciences and Engineering Research Council (NSERC) for a Discovery grant to R.H.M. and for a Canadian Graduate Scholarship and a Vanier Scholarship for J.F.S.. The authors wish to acknowledge the Canadian Foundation for Innovation, project number 19119, and the Ontario Research Fund for funding of the Centre for Spectroscopic Investigation of Complex Organic Molecules and Polymers. 


\section{References}

1. a) Blaser HU, Malan C, Pugin B, Spindler F, Steiner H, Studer M (2003) Adv. Synth. Catal. 345: 103. ; b) Naud F, Spindler F, Rueggeberg CJ, Schmidt AT, Blaser HU (2007) Org. Process Res. Dev. 11: 519. ; c) Pugin B, Blaser HU (2010) Top. Catal. 53: 953.

2. Ikariya T, Murata K, Noyori R (2006) Org. Biomol. Chem. 4: 393.

3. a) Samec JSM, Bäckvall JE, Andersson PG, Brandt P (2006) Chem. Soc. Rev. 35: 237.;

b) Noyori R, Ohkuma T (2001) Angew. Chem. Int. Ed. 40: 40.; c) Noyori R (2002) Angew. Chem. Int. Ed. 41: 2008.

4. a) Vedejs E, Jure M (2005) Angew. Chem. Int. Ed. 44: 3974.; b) Pellissier H (2011) Adv. Syn. \& Catal. 353: 1613.

5. Hashiguchi S, Fujii A, Haack K-J, Matsumura K, Ikariya T, Noyori R (1997) Angew. Chem. Int. Ed. 36: 288.

6. a) Morris RH (2009) Chem. Soc. Rev. 38: 2282. ; b) Bolm C, Legros J, Le Paih J, Zani L (2004) Chem. Rev. 104: 6217.; c) Enthaler S, Junge K, Beller M (2008) Angew. Chem. Int. Ed. 47: 3317.

7. a) Meyer N, Lough AJ, Morris RH (2009) Chem. Eur. J. 15: 5605.; b) Mikhailine A, Lough AJ, Morris RH (2009) J. Am. Chem. Soc. 131: 1394.; c) Lagaditis PO, Lough AJ, Morris RH (2011) J. Am. Chem. Soc 133: 9662.; d) Sues PE, Lough AJ, Morris RH (2011) Organometallics 30: 4418.

8. Sonnenberg JF, Coombs N, Dube PA, Morris RH (2012) J. Am. Chem. Soc. 134: 5893.

9. Lagaditis PO, Lough AJ, Morris RH (2010) Inorg. Chem. 49: 10057.

10. a) Gao J-X, Ikariya T, Noyori R (1996) Organometallics 15: 1087.; b) Koike T, Murata K, Ikariya T (2000) Org. Lett. 2: 3833.; c) Ito M, Osaku A, Kitahara S, Hirakawa M, Ikariya T (2003) Tetrahedron Lett. 44: 7521.; d) Csjernyik G, Bogár K, Bäckvall J-E (2004) Tetrahedron Lett. 45: 6799.

11. a) Arita S, Koike T, Kayaki Y, Ikariya T (2008) Angew. Chem. Int. Ed. 47: 2447.; b) Wills M (2008) Angew. Chem. Int. Ed. 47: 4264.

12. Bagdanoff JT, Stoltz BM (2004) Angew. Chem. Int. Ed. 43: 353.

13. Kumar Alamsetti S, Muthupandi P, Sekar G (2009) Chem. Eur. J. 15: 5424.

14. Alamsetti SK, Mannam S, Mutupandi P, Sekar G (2009) Chem. Eur. J. 15: 1086.

15. a) Muthupandi P, Alamsetti SK, Sekar G (2009) Chem. Commun. 3288; b) Kunisu T, Oguma T, Katsuki T (2011) J. Am. Chem. Soc 133: 12937. 
16. Martin VS, Woodard SS, Katsuki T, Yamada Y, Ikeda M, Sharpless KB (1981) J. Am. Chem. Soc 103: 6237.

17. a) Crabtree RH (2012) Chem. Rev. 112: 1536.; b) Widegren JA, Finke RG (2003) J. Mol. Catal. A: Chem. 198: 317.

18. Phua PH, Lefort L, Boogers JAF, Tristany M, de Vries JG (2009) Chem. Commun. 3747.

19. Mikhailine AA, Maishan MI, Lough AJ, Morris RH (2012) J. Am. Chem. Soc. 134: 12266.

20. a) Rangheard C, de Julian Fernandez C, Phua PH, Hoorn J, Lefort L, de Vries JG (2010) Dalton Trans. 39: 8464.; b) Clark TJ, Jaska CA, Turak A, Lough AJ, Lu ZH, Manners I (2007) Inorg. Chem. 46: 7394.; c) Jaska CA, Temple K, Lough AJ, Manners I (2003) J. Am. Chem. Soc. 125: 9424.; d) Hornstein BJ, Aiken JD, Finke RG (2002) Inorg. Chem. 41: 1625 .

21. Witham CA, Huang W, Tsung C-K, Kuhn JN, Somorjai GA, Toste FD (2010) Nat. Chem. 2: 36 .

22. Keifer PA, Baltusis L, Rice DM, Tymiak AA, Shoolery JN (1996) J. Magn. Reson., Ser. A 119: 65.

23. Santini R, Griffith MC, Qi M (1998) Tetrahedron Lett. 39: 8951.

\section{Table of Contents Graphic}

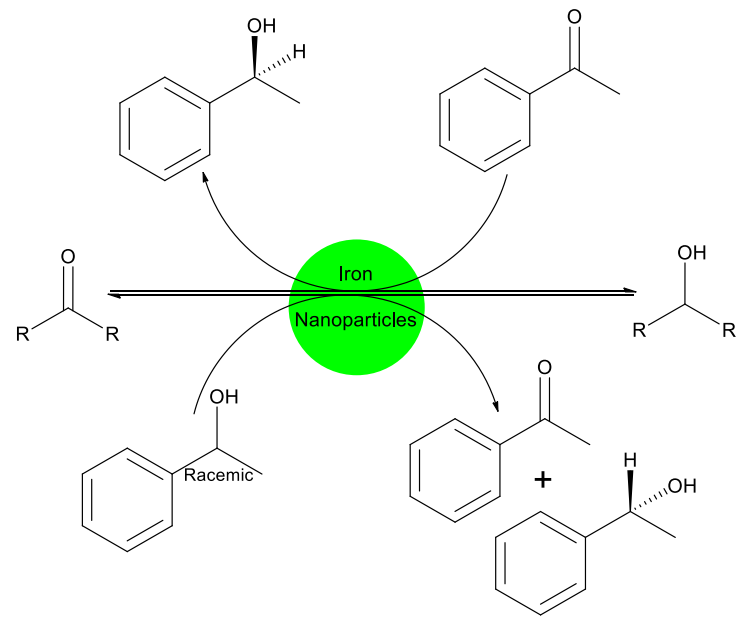




\section{Supporting Information: \\ Oxidative Kinetic Resolution of Aromatic Alcohols using Iron Nanoparticles}

Jessica F. Sonnenberg, Dmitry Pichugin, Neil Coombs and Robert H. Morris*

Lash Miller Laboratories, Department of Chemistry, University of Toronto, 80 St. George Street, Toronto, Ontario, M5S 3H6

\section{Table of Contents}

1) Experimental Section

2) 1-Phenylethanol Oxidation Runs

3) Oxidation Runs with Other Substrates

4) Poisoning Experiments

5) Initiation Period Experiments

6) Electron Microscopy Imaging

7) Polymer-Supported Substrate Experiments-Experimental and NMR plots

8) References

\section{Experimental Section}

\section{General Procedures}

All preparations, manipulations and catalysis were carried out under argon or nitrogen atmosphere using standard Schlenk line and drybox techniques. Dry and oxygen-free solvents and substrates were distilled and dried using the appropriate drying agents. NMR solvents were purchased from Aldrich and degassed and dried over activated molecular sieves. All other reagents were purchased from various commercial sources and used without further purification. NMR spectra were recorded using a Bruker 400 and Varian 300 and 400 spectrometers to determine ${ }^{1} \mathrm{H}(400$ and $300 \mathrm{MHz})$ and ${ }^{31} \mathrm{P}\left\{{ }^{1} \mathrm{H}\right\}(121 \mathrm{MHz})$ shifts. ${ }^{1} \mathrm{H}$ shifts are referenced to deuterated solvents, and ${ }^{31} \mathrm{P}$ peaks are externally referenced to $85 \%$ phosphoric acid. Solid state MAS NMR setup parameters are outlined in the 'Polymer-Supported Substrate Experiments' section.

\section{Gas Chromatography}

Gas Chromatography was done on a Perkin Elmer Clarus 400 Chromatograph equipped with a chiral column (CP chirasil-Dex CB $25 \mathrm{~m} \times 2.5 \mathrm{~mm}$ ) and auto-sampling capabilities. Hydrogen gas was used as 
the mobile phase, and the oven temperature was varied dependent on the substrate, as shown in Table 1.

Table 1: GC temperatures and retention times for substrates tested

\section{Ketone $\quad$ Alcohol Oven Temp $\left({ }^{\circ} \mathrm{C}\right) \quad \mathbf{t}_{\mathbf{k}}(\min ) \quad \mathbf{t}_{\mathbf{a}}(\min )$}<smiles>CC(=O)c1ccccc1</smiles><smiles>CC(O)c1ccccc1</smiles>

130

4.56

$7.58,8.03$<smiles>CCC(=O)c1ccccc1</smiles><smiles>CCC(O)c1ccccc1</smiles>

120

9.59

$20.74,21.94$<smiles>CC(=O)CCc1ccccc1</smiles><smiles>CC(O)CCc1ccccc1</smiles>

105

30.67<smiles>CC(=O)c1cccc(Cl)c1</smiles><smiles>CC(O)c1cccc(Cl)c1</smiles>

140

6.46

$14.81,16.05$<smiles>CC(=O)c1ccc(Cl)cc1</smiles><smiles>CC(O)c1ccc(Cl)cc1</smiles>

145

5.96

$11.03,12.09$<smiles>CC(C)C(=O)c1ccccc1</smiles><smiles>CC(C)C(O)c1ccccc1</smiles>

140

5.19

10.06 (rac)<smiles>CC(C)(C)C(=O)c1ccccc1</smiles><smiles>CC(C)(C)C(O)c1ccccc1</smiles>

140

5.67

$13.15,13.78$<smiles>CC(=O)c1ccc(C)cc1</smiles><smiles>Cc1ccc(C(C)O)cc1</smiles>

135

6.40

$9.11,10.04$

\section{Synthesis}

Precatalyst $\left[\mathrm{Fe}(\mathrm{CO})(\mathrm{NCMe})\left(\mathrm{P}_{2} \mathrm{~N}_{2}\right.\right.$ dpen $\left.)\right]\left[\mathrm{BF}_{4}\right]_{2}(2)$ has been prepared and characterised previously. ${ }^{1}$

\section{Microscopy}

Electron microscopy images were carried out at the 'Centre for Nanostructure Imaging' (University of Toronto) in collaboration with Dr. Neil Coombs, on a Hitachi HD-2000 STEM operating at $-100^{\circ} \mathrm{C}$. Low temperatures were required to minimize contamination and specimen damage by the electron beam. Samples were placed on an ultrathin carbon film supported by a lacey carbon film on a 400 mesh copper 
grid. Energy-Dispersive X-Ray Spectroscopy (EDX) was run concurrently using INCA software and the STEM. Sample preparation: to a vial containing pre-catalyst $(6 \mathrm{mg}, 0.0057 \mathrm{mmol})$ and base, solvent and substrate were added at the desired temperature, in an argon filled glovebox. The substrate used was iPrOH ( $0.1 \mathrm{~mL}, 1.3 \mathrm{mmol}$ ) as it has a low boiling point and could be easily evaporated off the grids under vacuum before analysis. For reactions employing benzophenone as the sacrificial oxidant, benzophenone (1.4 g, $7.7 \mathrm{mmol}$ ) was dissolved in THF ( $4.7 \mathrm{~mL}, 58 \mathrm{mmol}$ ) and KOtBu ( $5 \mathrm{mg}, 0.045 \mathrm{mmol})$ was used as the base. Alternatively, acetone $(6 \mathrm{~mL}, 82 \mathrm{mmol}$ ) was used as the solvent with $\mathrm{NaOiPr}(4$ $\mathrm{mg}, 0.049 \mathrm{mmol}$ ) as the base.

\section{Solid State NMR}

Solid State NMR experiments were conducted at the 'Centre for Spectroscopic Investigation of Complex Organic Molecules and Polymers' (University of Toronto) in collaboration with Dmitry Pichugin. Carbon spectra were acquired on Agilent DD2 600-MHz spectrometer with an Agilent 3.2-mm narrow bore HXY solids probe. The probe was used in double-resonance mode; proton and carbon. Spectra were acquired using magic angle spinning(MAS) at $10-12 \mathrm{kHz}$ spinning using 'onepul' pulse sequence with calibrated 90 degree pulse, 2 second recycle delay, $50.8 \mathrm{~ms}$ acquisition time (2048 points), and spin decoupling. Experiment time was 4 hours with 7000 scans. $3.2 \mathrm{~mm}$ rotors were packed under air with $26.7 \mathrm{mg}$ of pre-swollen polymer beads (swollen using $\mathrm{CD}_{2} \mathrm{Cl}_{2}$ ), and pre-spun at $10 \mathrm{kHz}$ outside of the probe for 20 minutes prior to spinning in the NMR probe.

\section{Catalysis - Standard Run}

To a vial containing pre-catalyst ( $6 \mathrm{mg}, 0.0057 \mathrm{mmol})$ and $\mathrm{KOtBu}(5 \mathrm{mg}, 0.045 \mathrm{mmol})$, solvent and substrate were added at the desired temperature, in an argon filled glovebox. For reactions employing benzophenone as the sacrificial oxidant, benzophenone $(1.4 \mathrm{~g}, 7.7 \mathrm{mmol})$ was dissolved in THF $(4.7 \mathrm{~mL}$, $58 \mathrm{mmol})$. Alternatively, acetone $(6 \mathrm{~mL}, 82 \mathrm{mmol}$ ) was used as the solvent. Immediately upon addition of solvent and substrate to the vial, a dark brown solution was formed. Solutions were stirred vigorously, and samples were taken from the mixture, quenched by exposure to air and analysed by gas chromatography. When the samples are exposed to air the solution turns yellow and the reaction stops immediately. The alcohol/ketone concentration does not change in these solutions, even after several days. All of the catalytic results were reproduced to ensure consistency.

\section{Catalysis - Poisoned Run}

Solutions were prepared, initiated and monitored as outlined above. $\mathrm{PMe}_{3}$ in toluene $(0.85 \mu \mathrm{mol}$ in 0.15 $\mathrm{mL} / 1.4 \mathrm{mmol}$ toluene) was added to the reaction when conversion to ketone was $10 \%$ (varies dependent on substrate and conditions employed). This is similar to techniques employed for transfer hydrogenation previously reported. ${ }^{2}$ 


\section{1-Phenylethanol Oxidation Runs}

Figure 1: Reaction profile for the catalytic oxidation of racemic 1-phenylethanol $(0.27 \mathrm{~mL}, 2.2 \mathrm{mmol})$ to acetophenone at room temperature $\left(28^{\circ} \mathrm{C}\right)$ in THF $(4.7 \mathrm{~mL})$ with benzophenone $(1.4 \mathrm{~g}, 7.7 \mathrm{mmol})$.

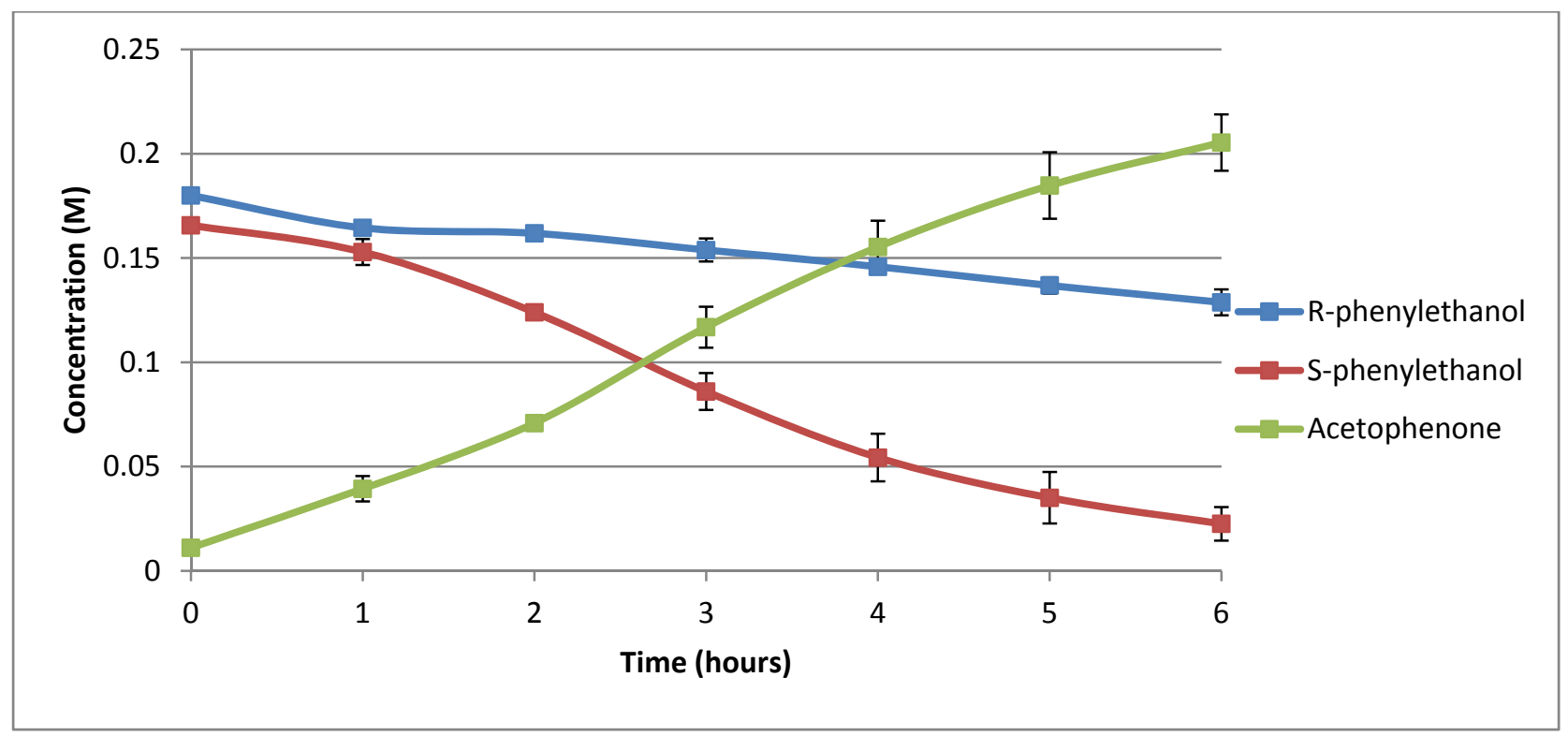

Figure 2: Enantiopurity profile for catalysis depicted in Figure 1

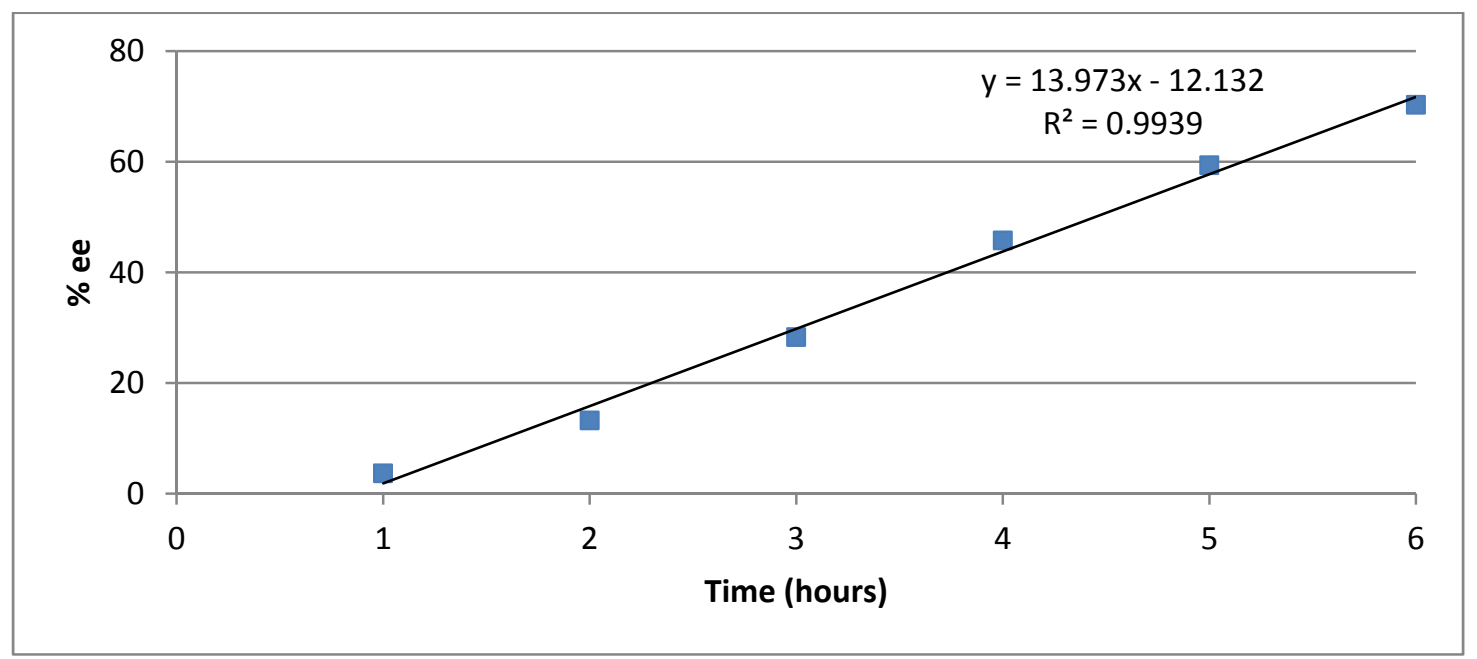


Figure 3: Reaction profile for the catalytic oxidation of racemic 1-phenylethanol $(0.27 \mathrm{~mL}, 2.2 \mathrm{mmol})$ to acetophenone at $45^{\circ} \mathrm{C}$ in THF ( $4.7 \mathrm{~mL})$ with benzophenone $(1.4 \mathrm{~g}, 7.7 \mathrm{mmol})$.

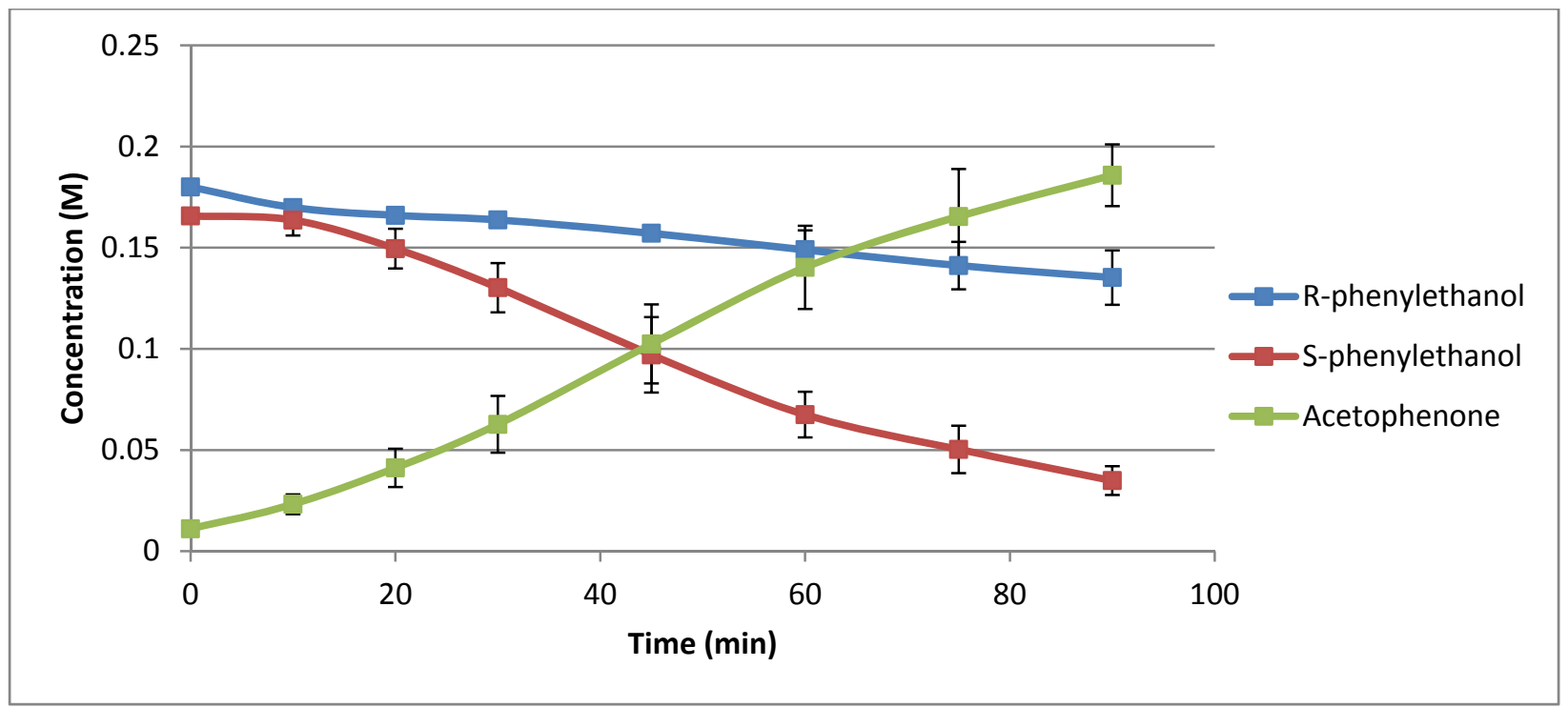

Figure 4: Enantiopurity profile for catalysis depicted in Figure 3.

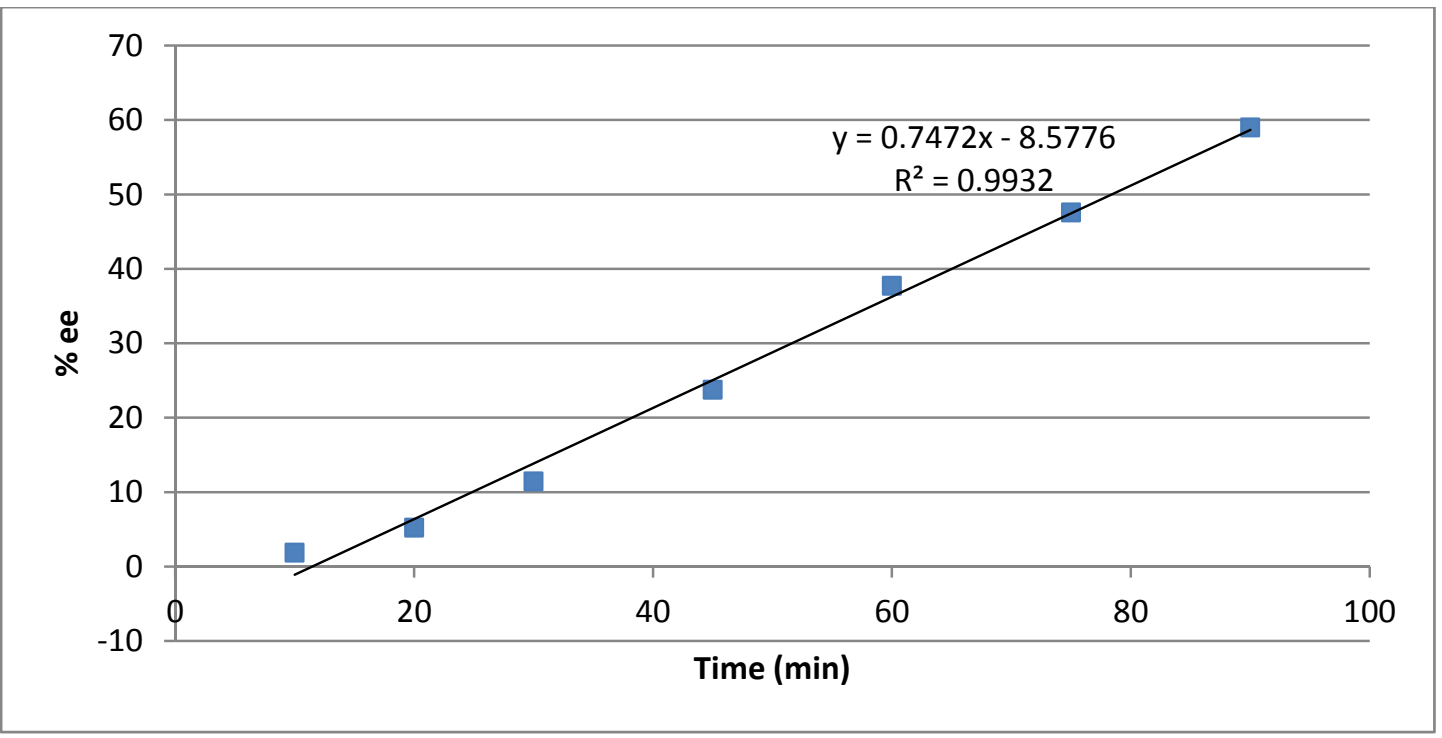


Figure 5: Reaction profile for the catalytic oxidation of racemic 1-phenylethanol $(0.27 \mathrm{~mL}, 2.2 \mathrm{mmol})$ to acetophenone at $45^{\circ} \mathrm{C}$ in THF ( $4.7 \mathrm{~mL}$ ) with benzophenone $(1.4 \mathrm{~g}, 7.7 \mathrm{mmol})$ using NaOiPr $(4 \mathrm{mg}, 0.049 \mathrm{mmol})$ as the base.

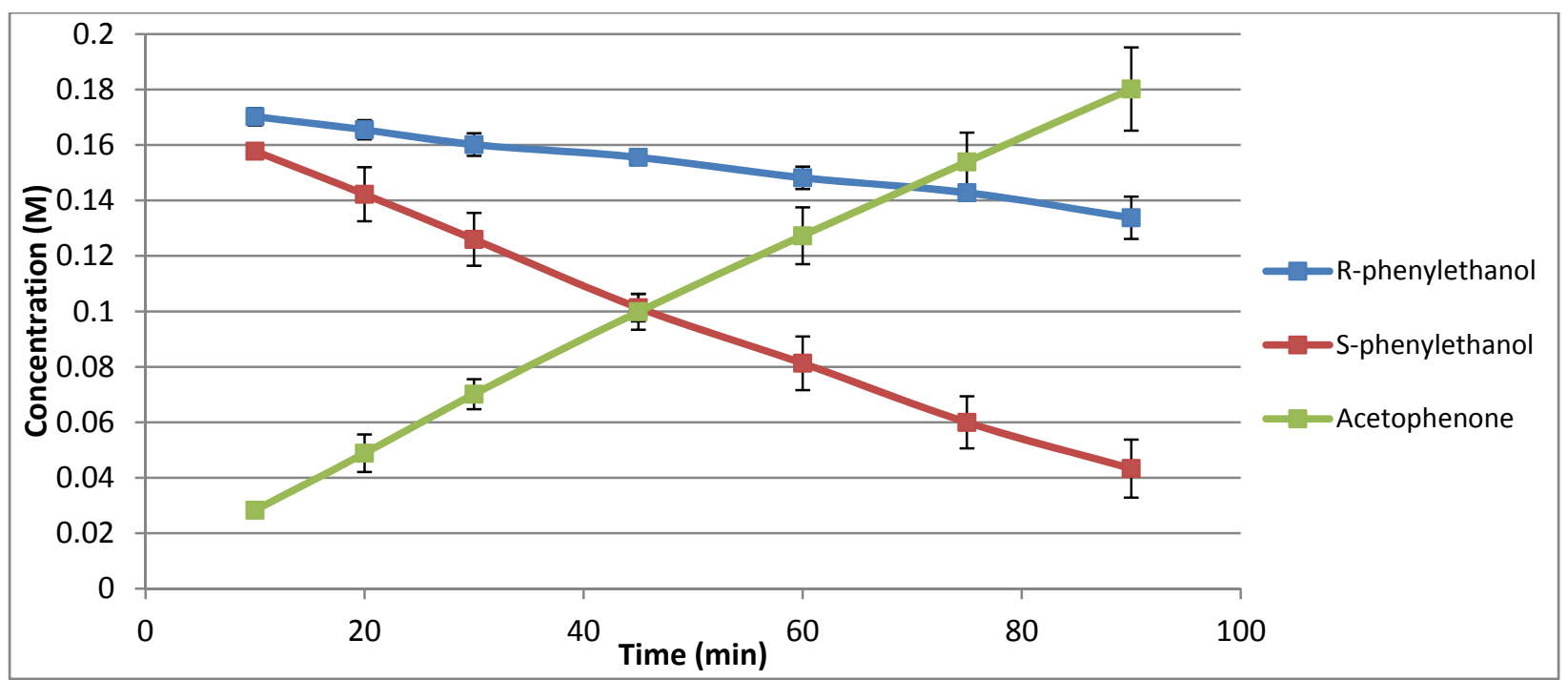

Figure 6: Enantiopurity profile for catalysis depicted in Figure 5.

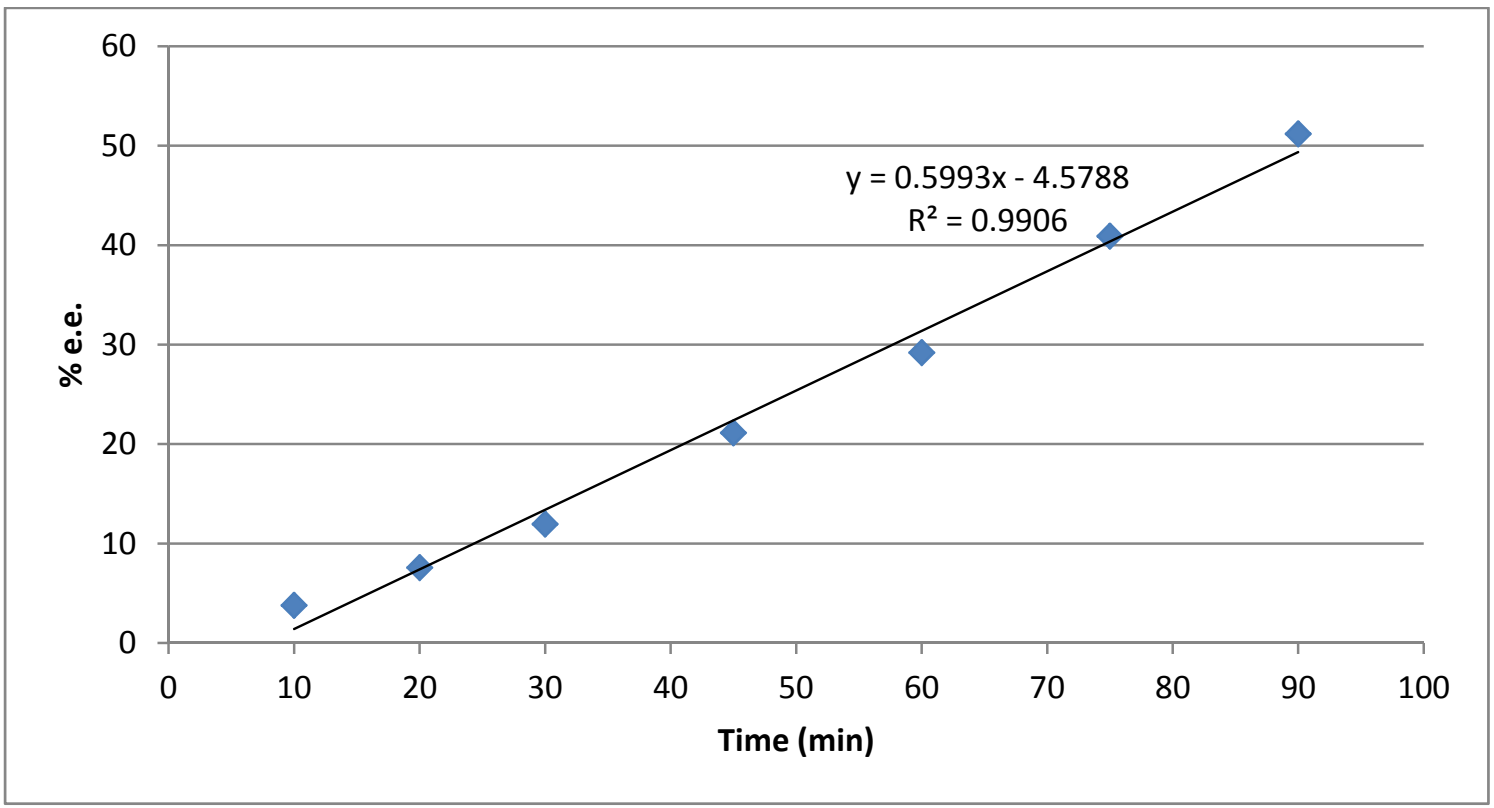


Figure 7: Reaction profile for the catalytic oxidation of racemic 1-phenylethanol $(0.27 \mathrm{~mL}, 2.2 \mathrm{mmol})$ to acetophenone at room temperature $\left(28^{\circ} \mathrm{C}\right)$ in acetone $(6 \mathrm{~mL})$.

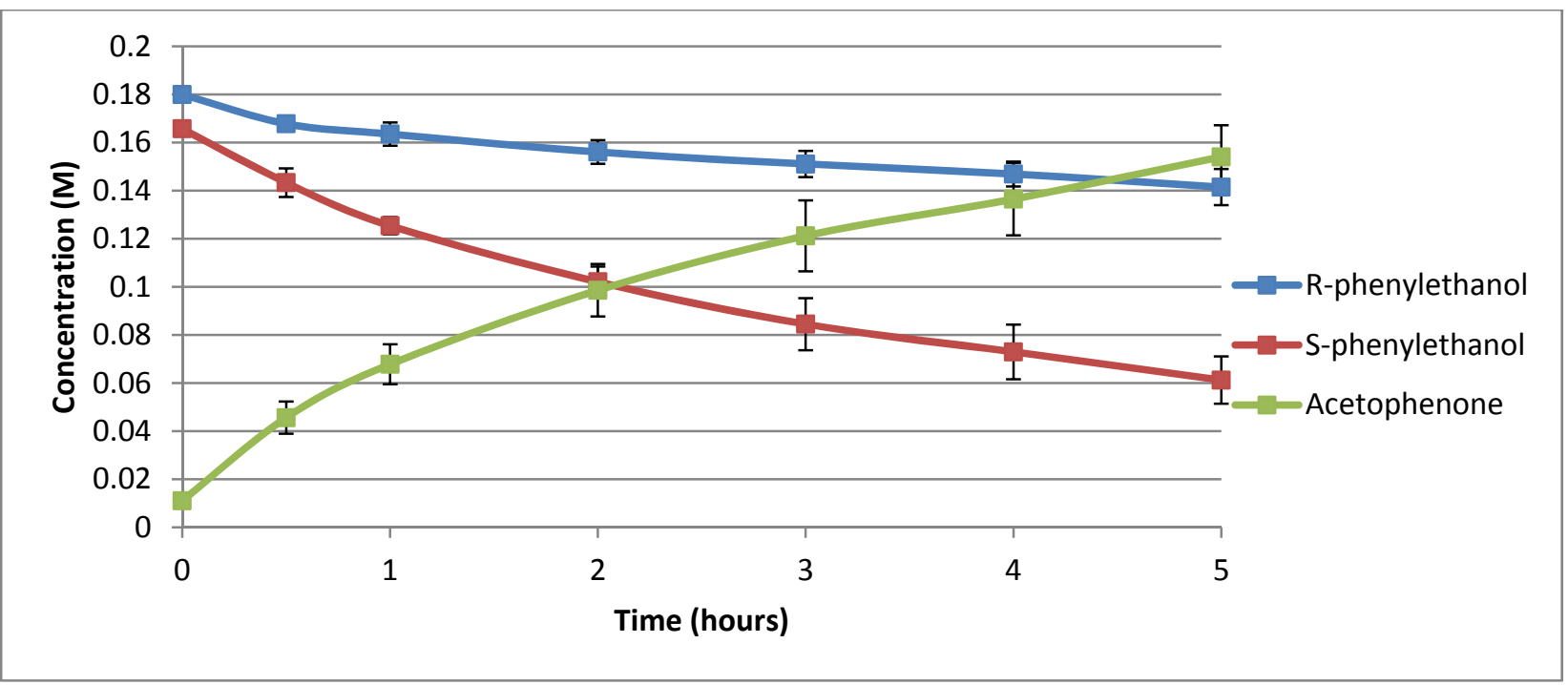

Figure 8: Enantiopurity profile for catalysis depicted in Figure 7.

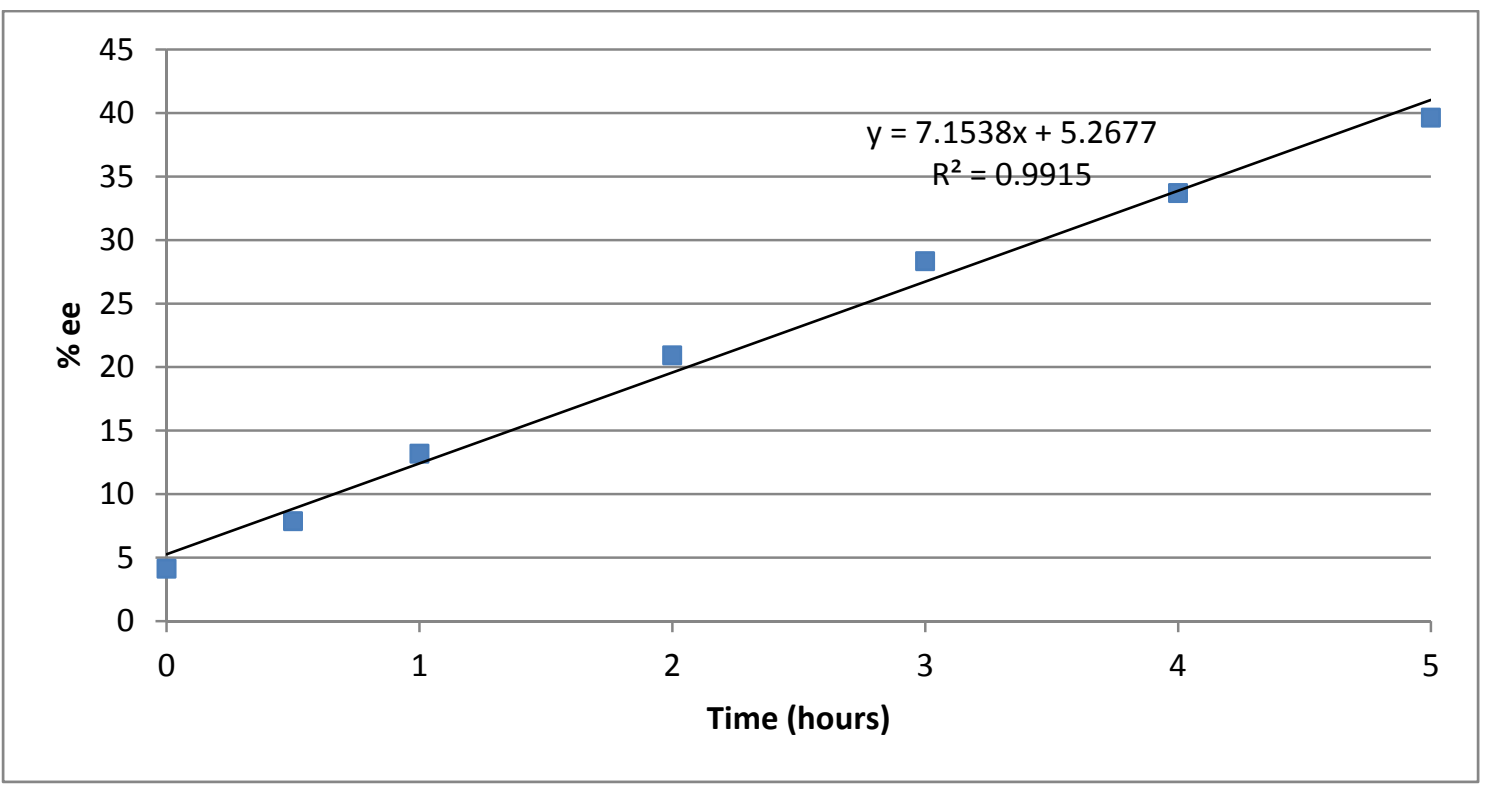


Figure 9: Reaction profile for the catalytic oxidation of racemic 1-phenylethanol $(0.27 \mathrm{~mL}, 2.2 \mathrm{mmol})$ to acetophenone at room temperature $\left(28^{\circ} \mathrm{C}\right)$ in acetone $(6 \mathrm{~mL})$ using NaOiPr $(4 \mathrm{mg}, 0.049 \mathrm{mmol})$.

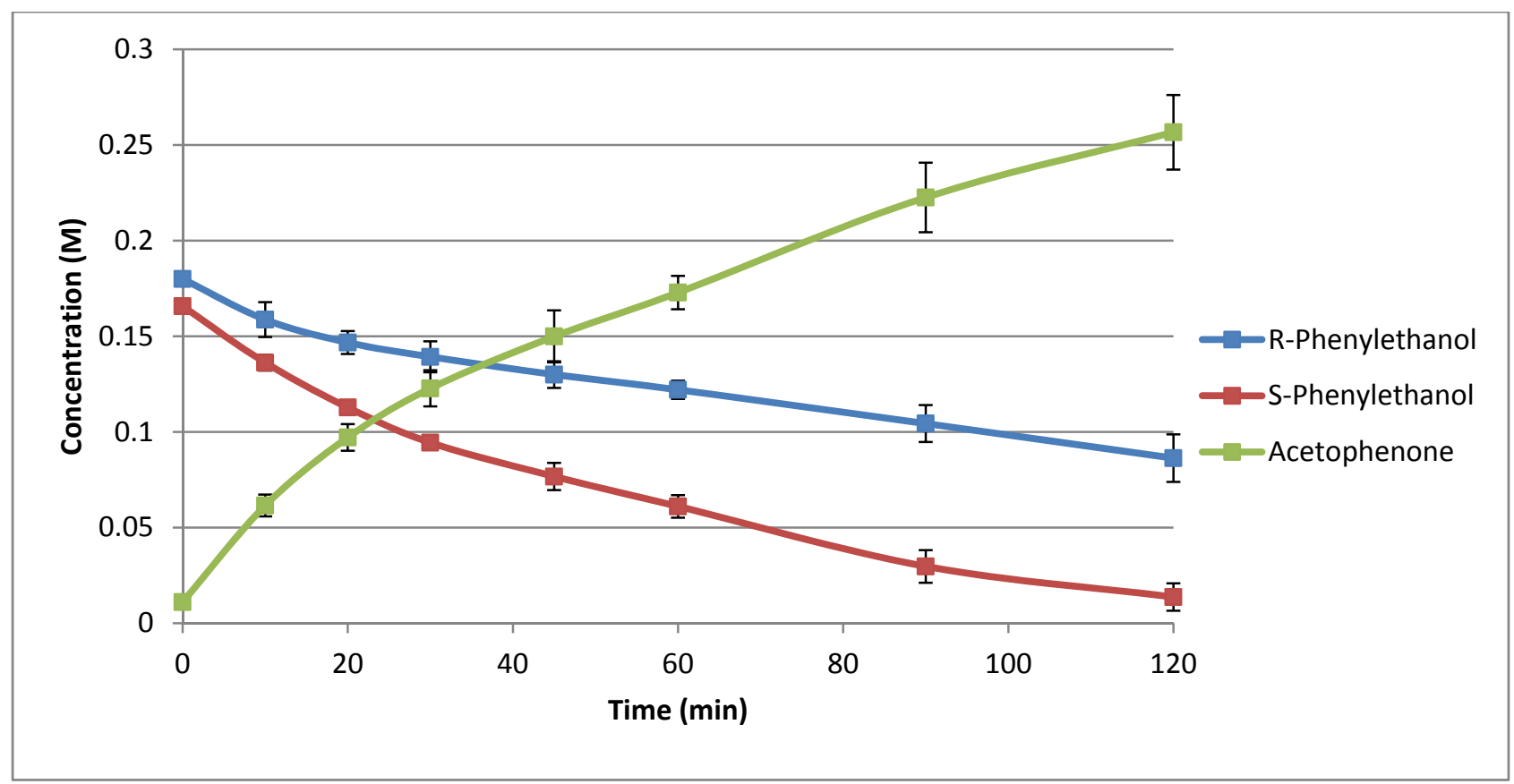

Figure 10: Enantiopurity profile for catalysis depicted in Figure 9.

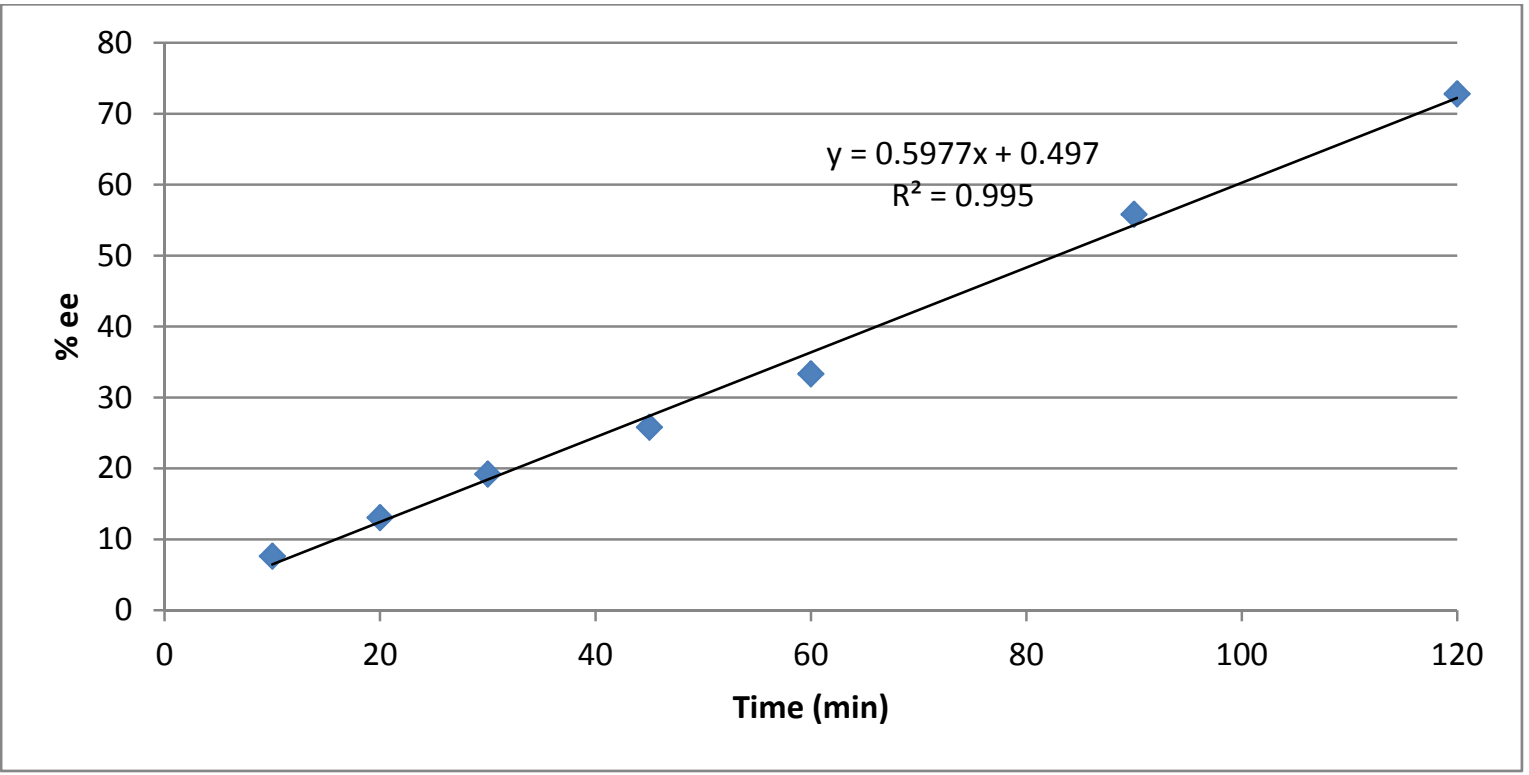




\section{Oxidation Runs with other Substrates}

Figure 11: Reaction profile for the catalytic oxidation of racemic 1-phenylpropanol $(0.31 \mathrm{~mL}, 2.3 \mathrm{mmol})$ to propiophenone at $45^{\circ} \mathrm{C}$ in THF (4.7 $\left.\mathrm{mL}\right)$ with benzophenone $(1.4 \mathrm{~g}, 7.7 \mathrm{mmol})$.

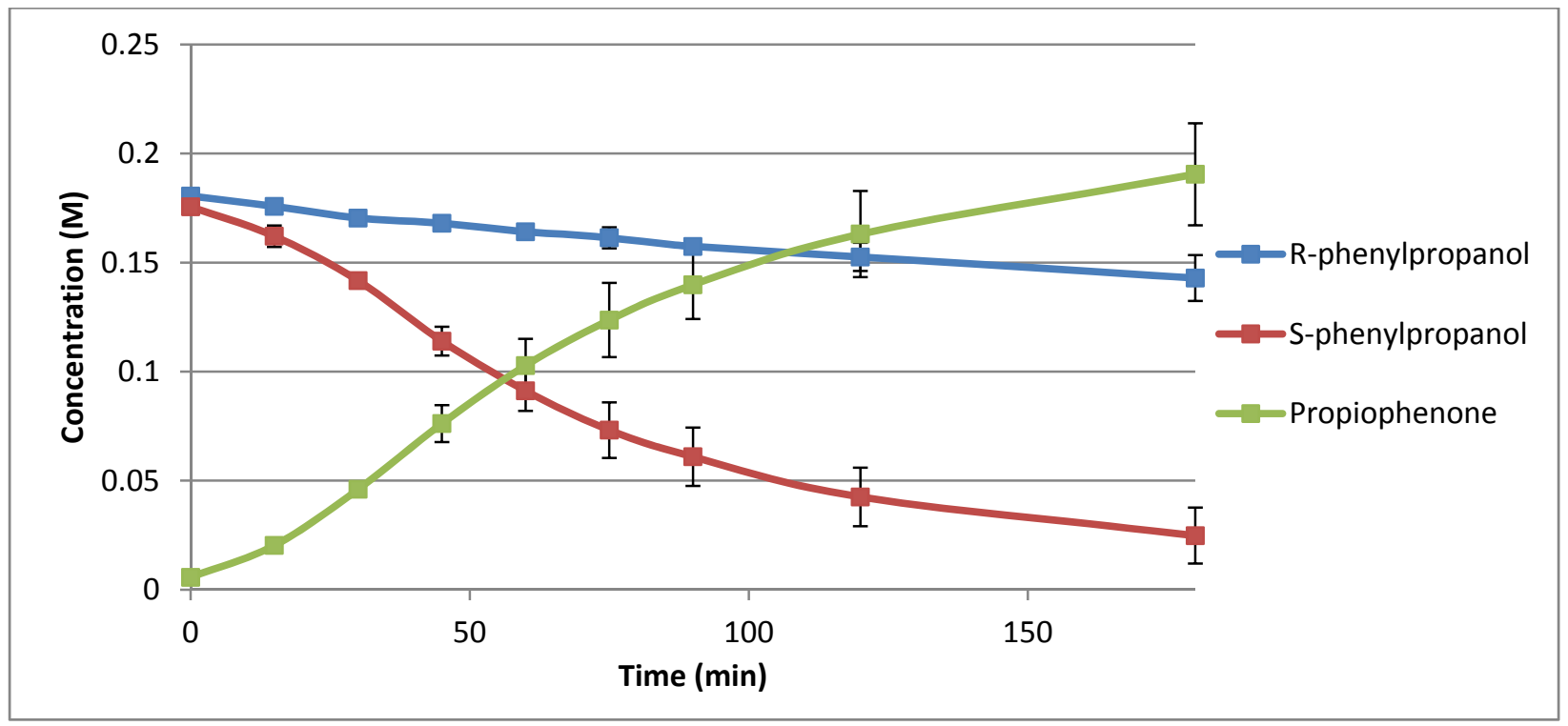

Figure 12: Enantiopurity profile for catalysis depicted in Figure 11.

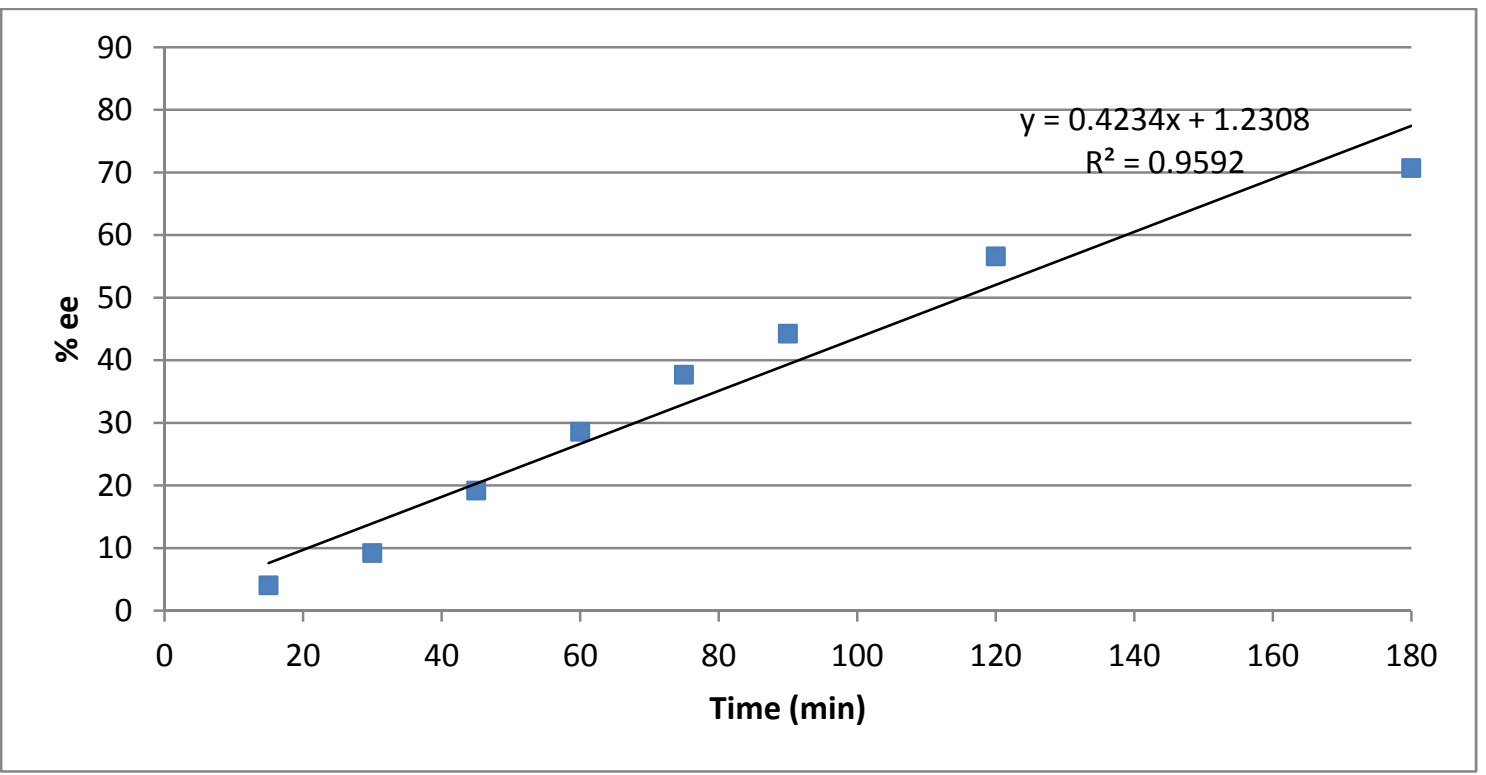


Figure 13: Reaction profile for the catalytic oxidation of racemic 4-phenyl-2-butanol $(0.10 \mathrm{~mL}, 0.65 \mathrm{mmol})$ to 4 -phenyl-2butanone at $45^{\circ} \mathrm{C}$ in THF $(4.7 \mathrm{~mL})$ with benzophenone $(1.4 \mathrm{~g}, 7.7 \mathrm{mmol})$.

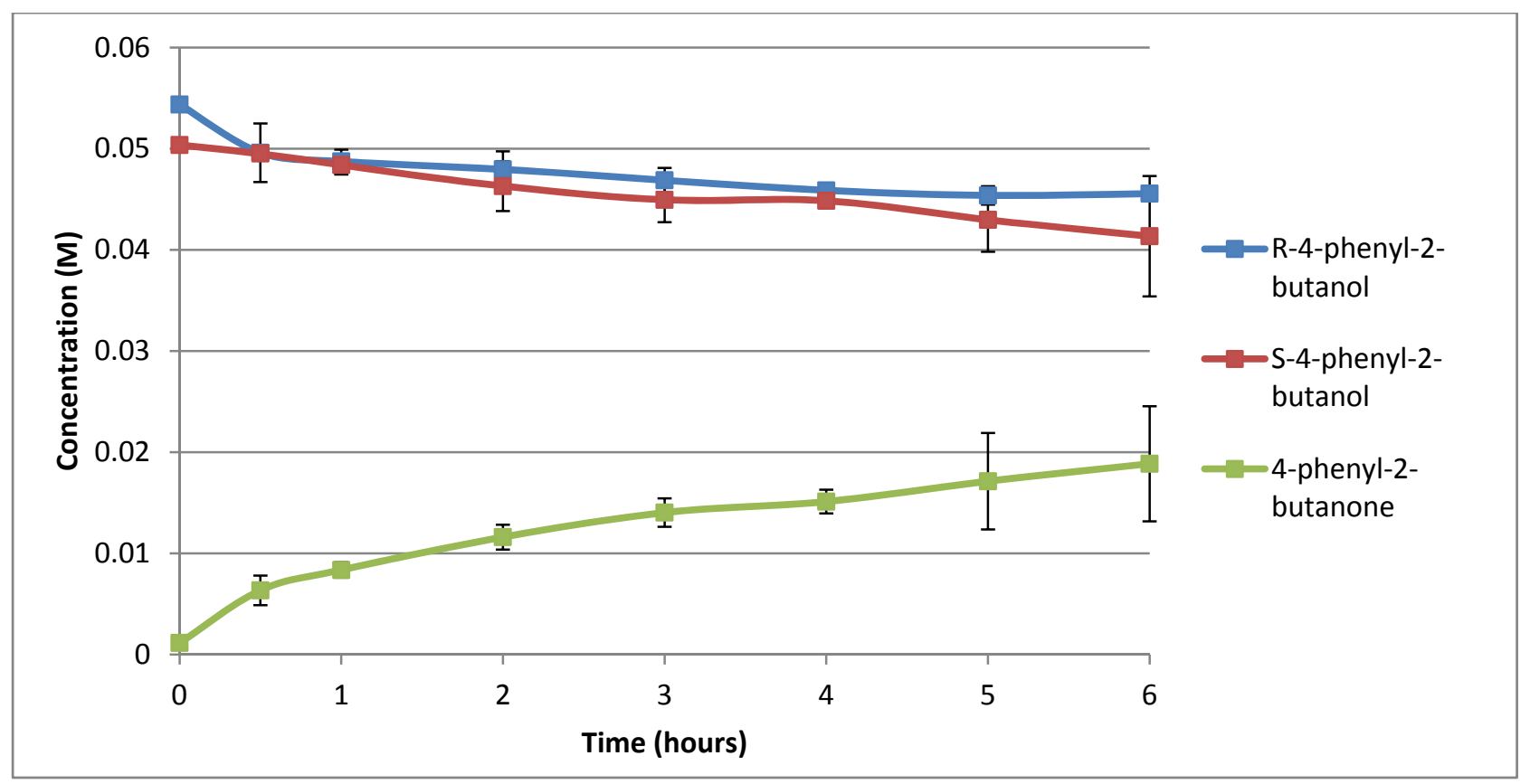

Figure 14: Enantiopurity profile for catalysis depicted in Figure 13.

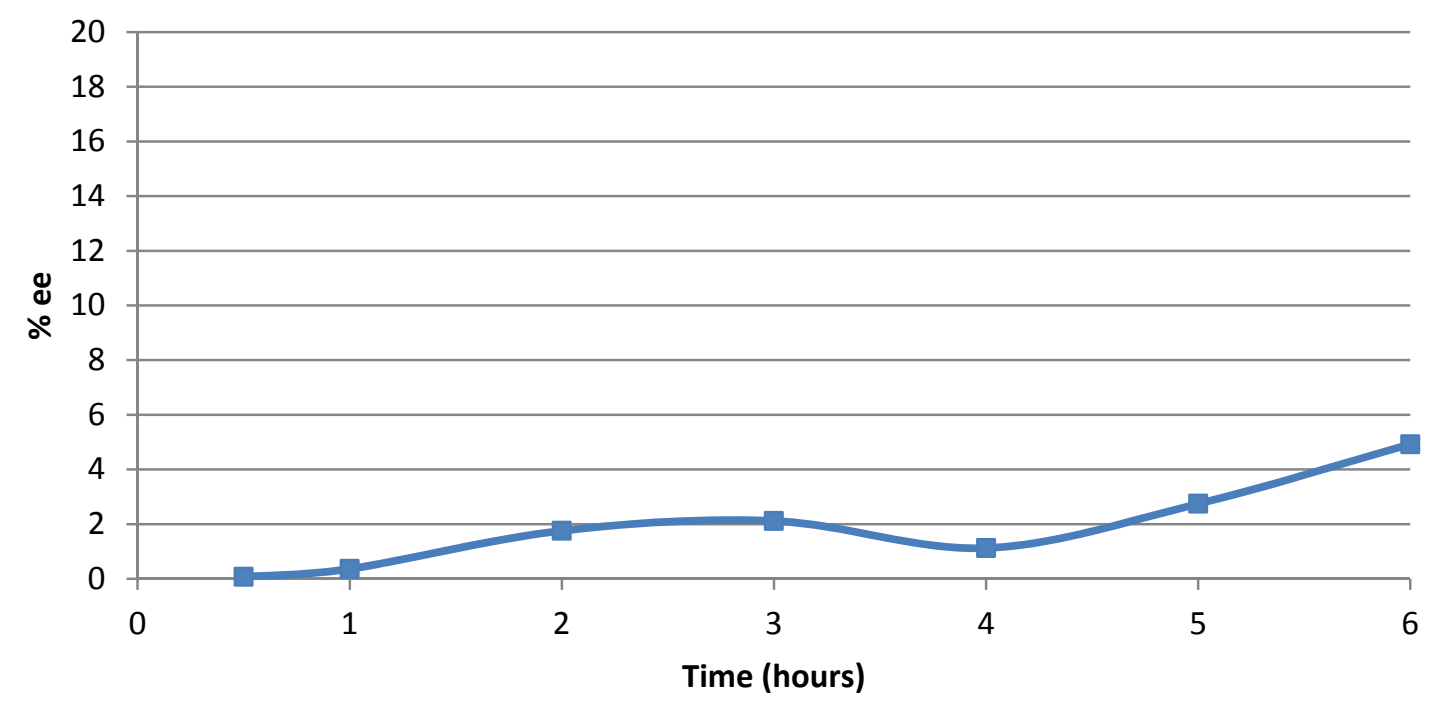


Figure 15: Reaction profile for the catalytic oxidation of racemic 4'-chlorophenylethanol (0.30 $\mathrm{mL}, 2.2 \mathrm{mmol})$ to $4^{\prime}$ chloroacetophenone at $45^{\circ} \mathrm{C}$ in THF $(4.7 \mathrm{~mL})$ with benzophenone $(1.4 \mathrm{~g}, 7.7 \mathrm{mmol})$.

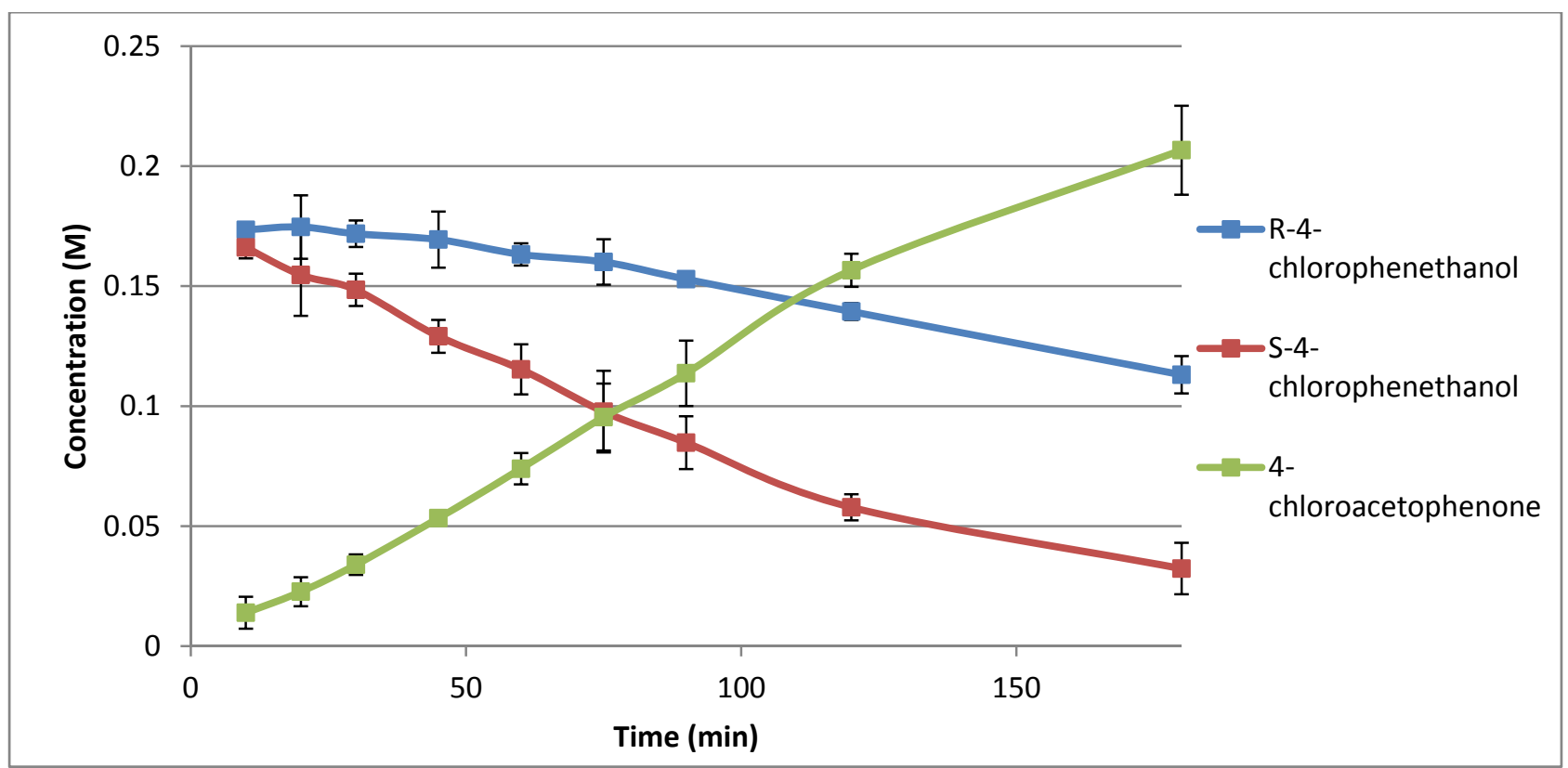

Figure 16: Enantiopurity profile for catalysis depicted in Figure 15.

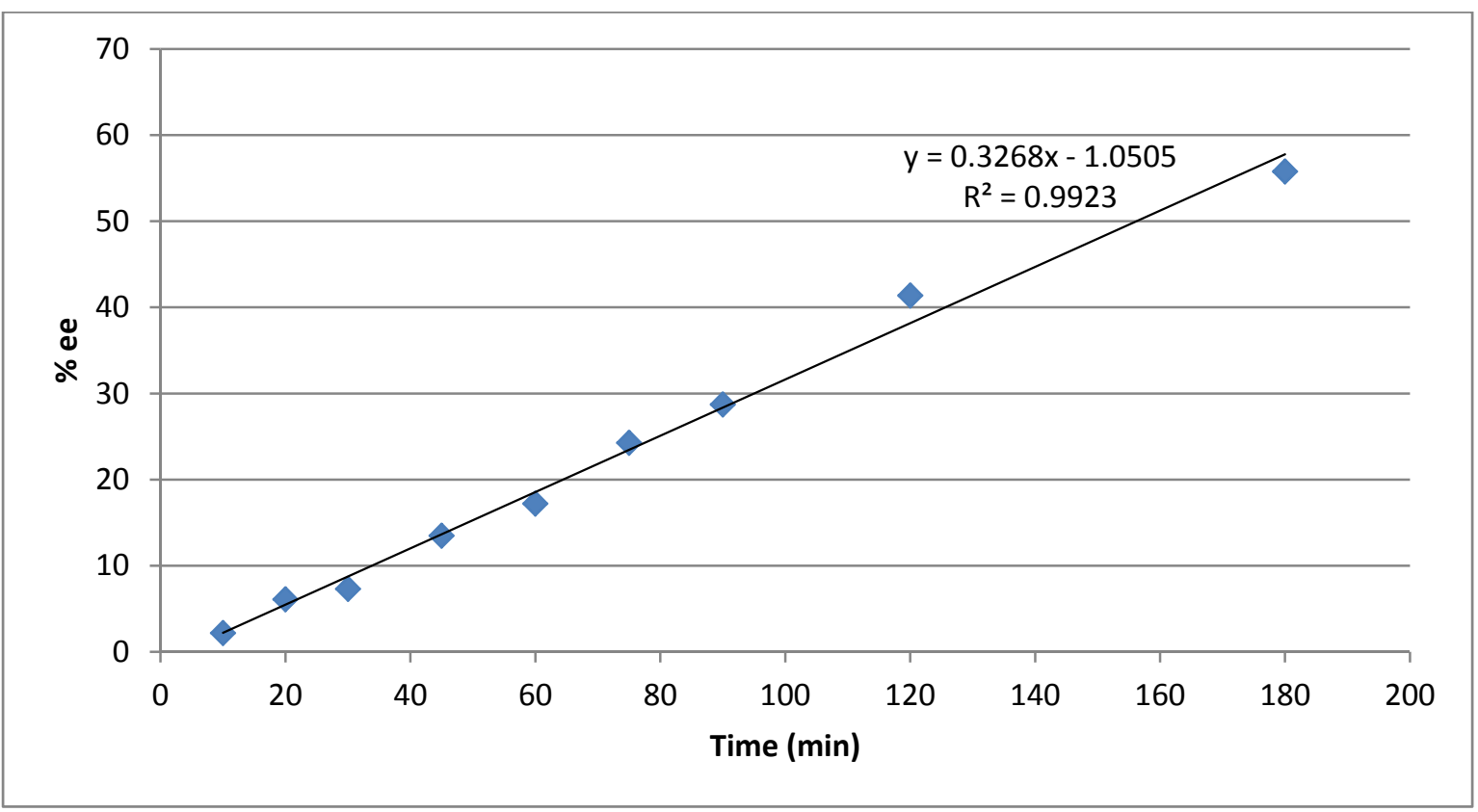


Figure 17: Reaction profile for the catalytic oxidation of racemic $3^{\prime}$-chlorophenylethanol (0.30 $\left.\mathrm{mL}, 2.2 \mathrm{mmol}\right)$ to $3^{\prime}$ chloroacetophenone at $45^{\circ} \mathrm{C}$ in THF $(4.7 \mathrm{~mL})$ with benzophenone $(1.4 \mathrm{~g}, 7.7 \mathrm{mmol})$.

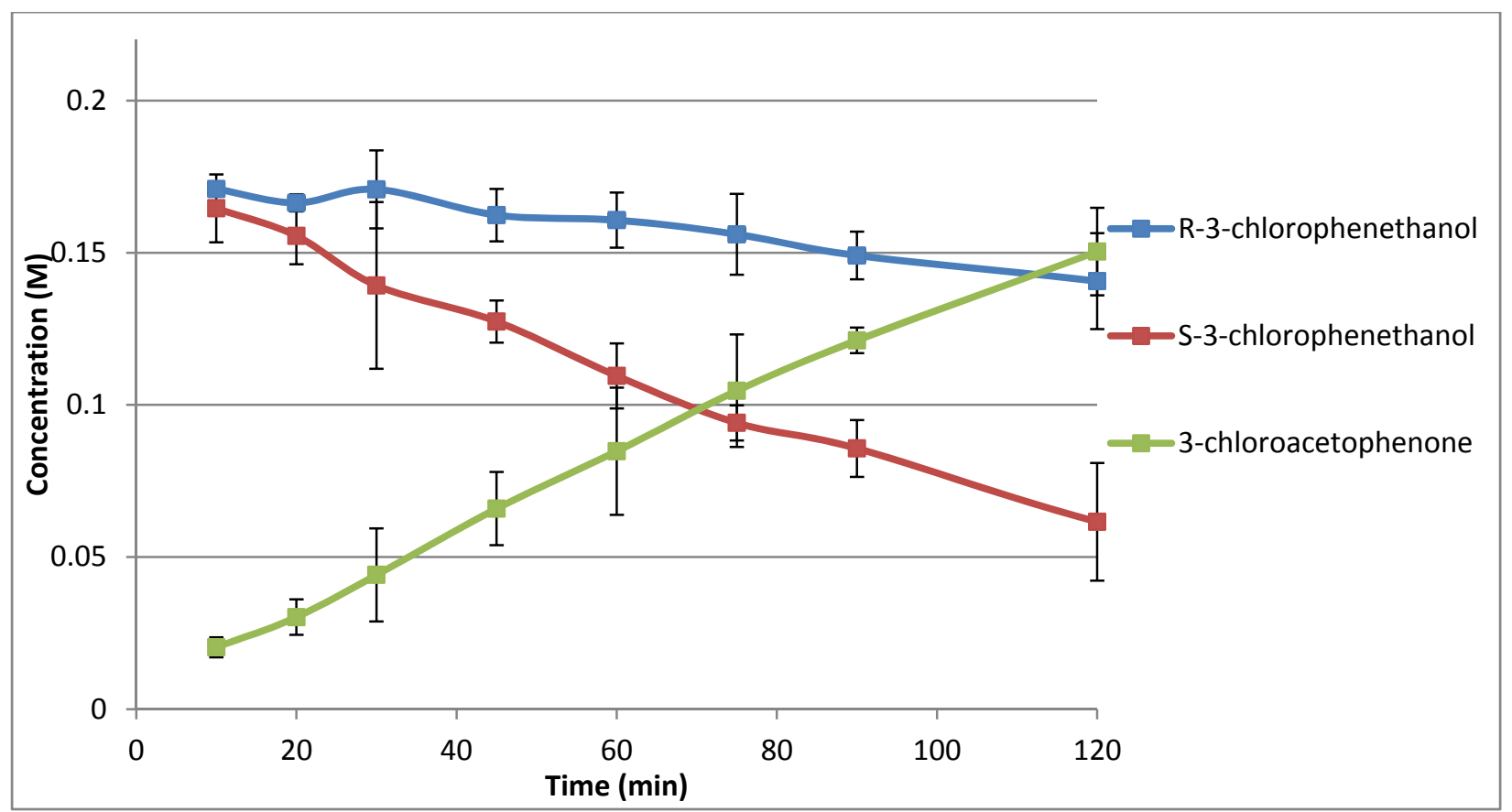

Figure 18: Enantiopurity profile for catalysis depicted in Figure 17.

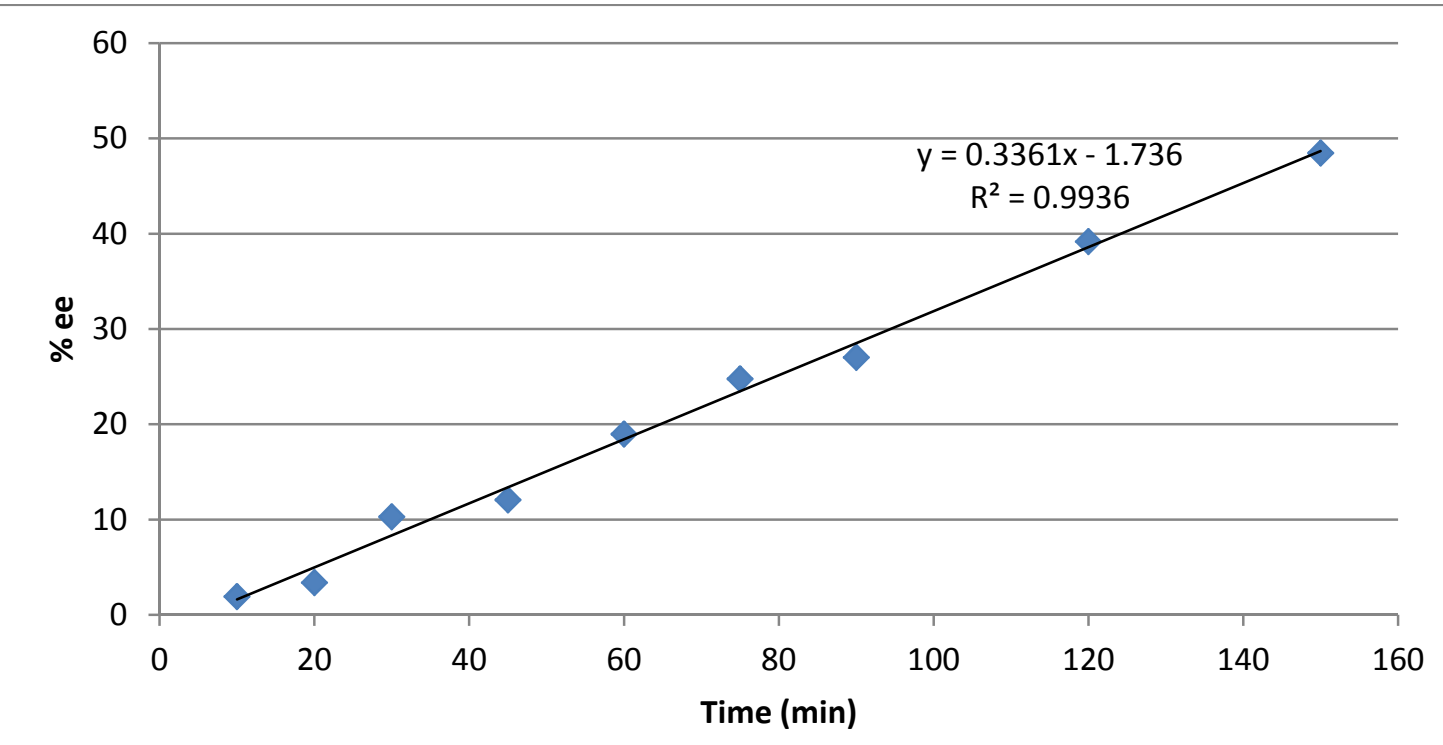


Figure 19: Reaction profile for the catalytic oxidation of racemic 2-methyl-1-phenylpropanol (0.26 $\mathrm{mL}, 1.7 \mathrm{mmol})$ to 2-methyl1-propiophenone at $45^{\circ} \mathrm{C}$ in THF $(4.7 \mathrm{~mL})$ with benzophenone $(1.4 \mathrm{~g}, 7.7 \mathrm{mmol})$.

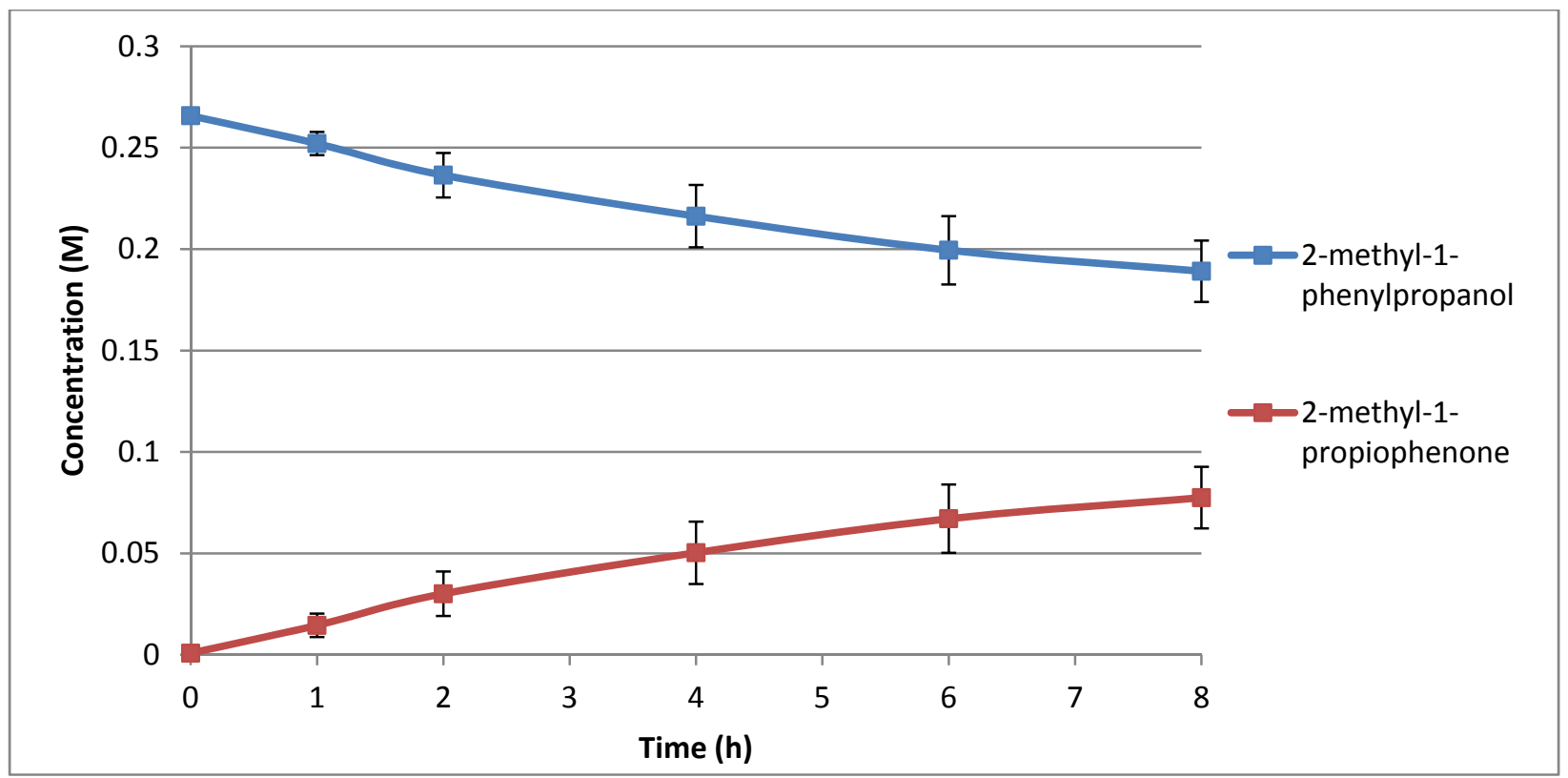

Figure 20: Reaction profile for the catalytic oxidation of racemic 4'-methylphenylethanol (0.31 mL, $2.2 \mathrm{mmol})$ to 4'methylacetophenone at $45^{\circ} \mathrm{C}$ in THF $(4.7 \mathrm{~mL})$ with benzophenone $(1.4 \mathrm{~g}, 7.7 \mathrm{mmol})$.

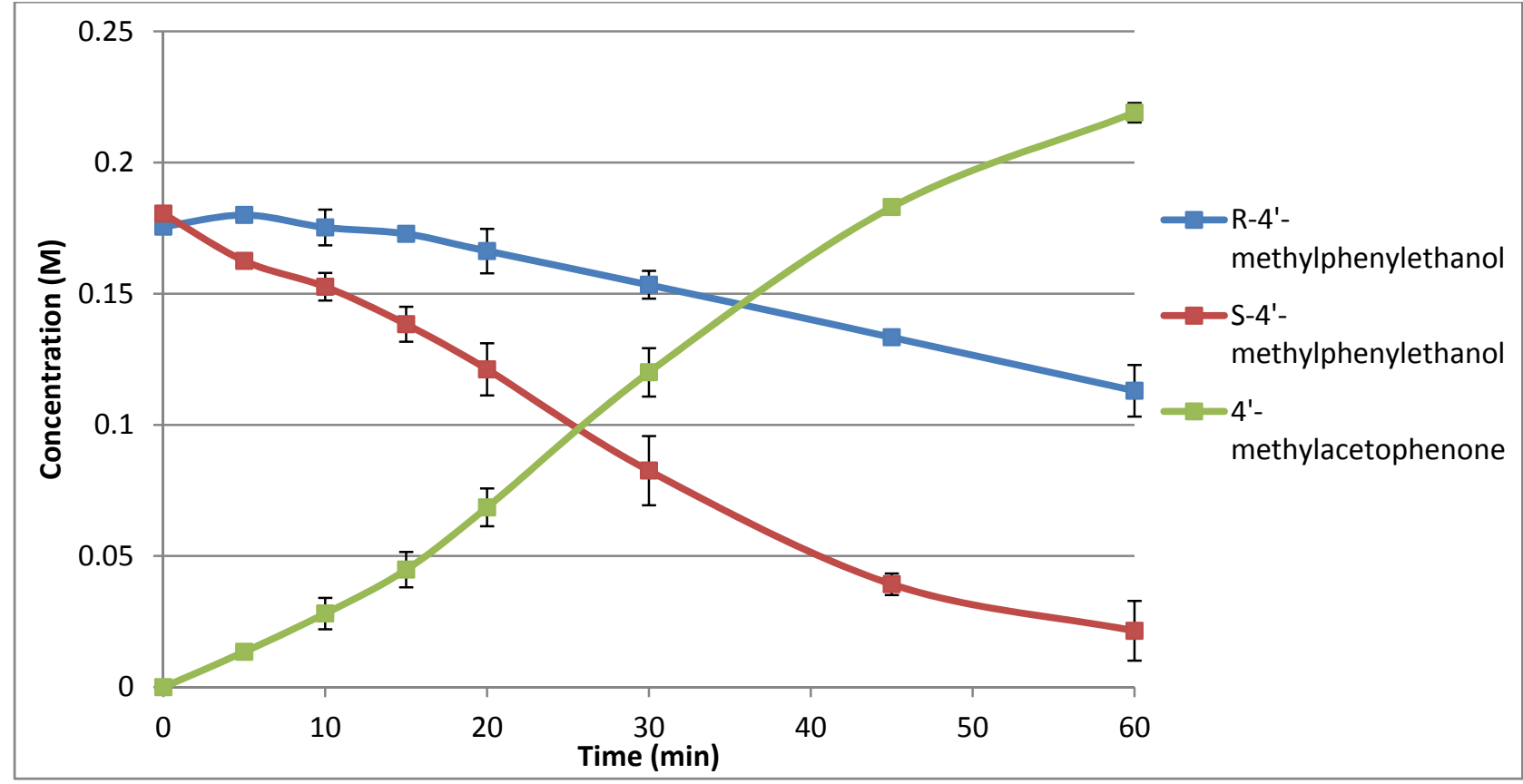


Figure 21: Enantiopurity profile for catalysis depicted in Figure 20.

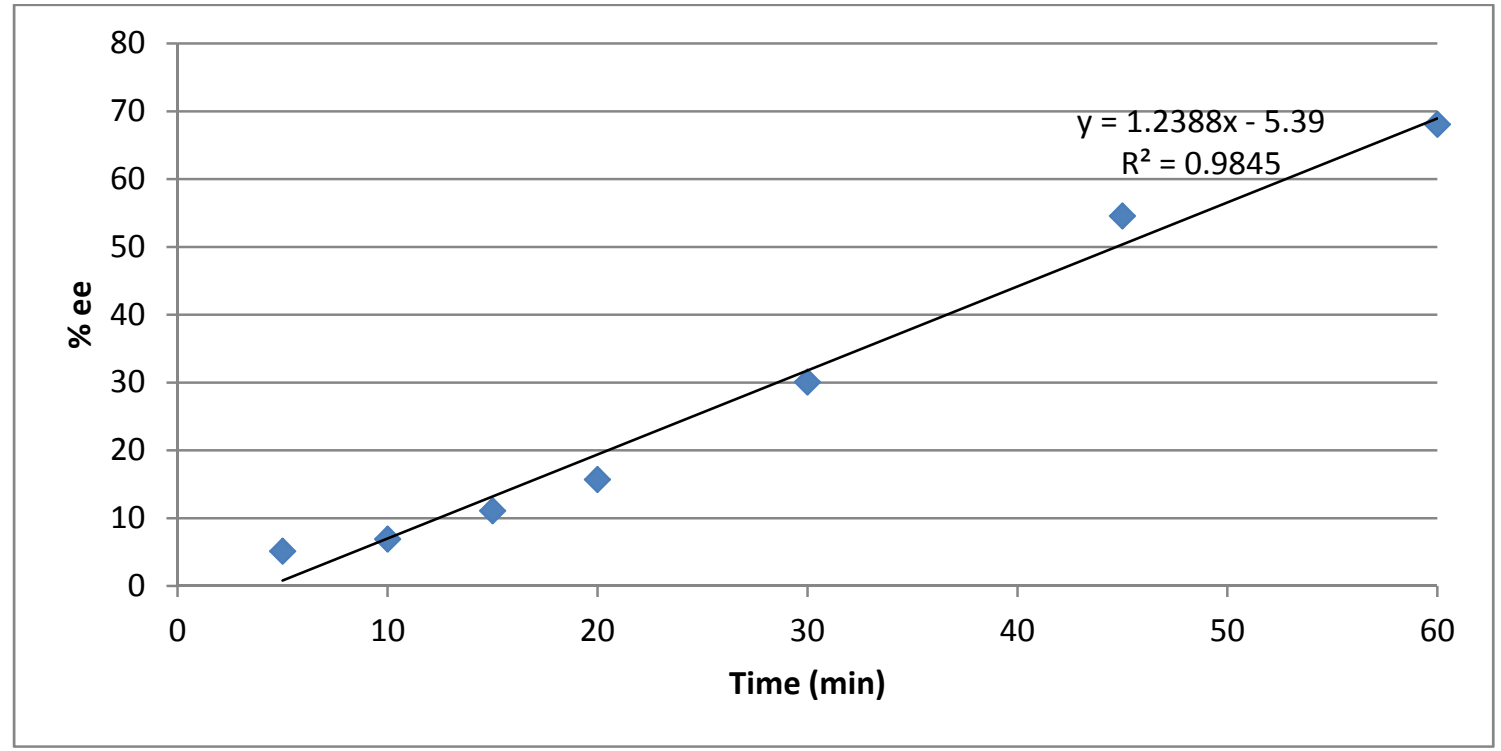

\section{Poisoning Experiments}

Figure 22: Reaction profiles for the catalytic oxidation of racemic 1-phenylethanol $(0.27 \mathrm{~mL}, 2.2 \mathrm{mmol})$ to acetophenone at room temperature $\left(28^{\circ} \mathrm{C}\right)$ in acetone $(6 \mathrm{~mL})$ using NaOiPr $(4 \mathrm{mg}, 0.049 \mathrm{mmol})$, and the poisoned runs using PMe $(0.85 \mu \mathrm{mol}$ in $0.15 \mathrm{~mL} / 1.4 \mathrm{mmol}$ toluene).

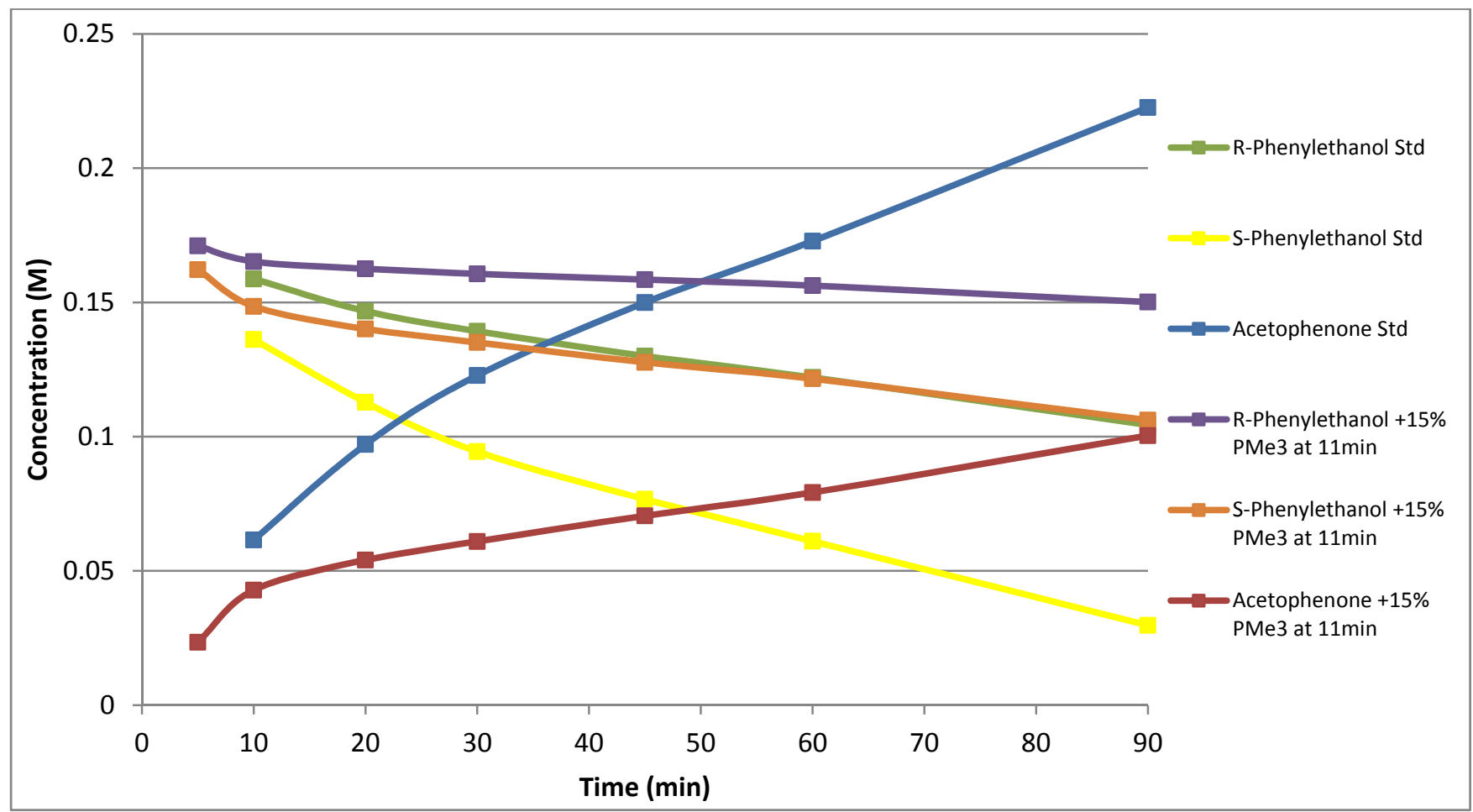


Figure 23: Enantiopurity profile for catalysis depicted in Figure 22.

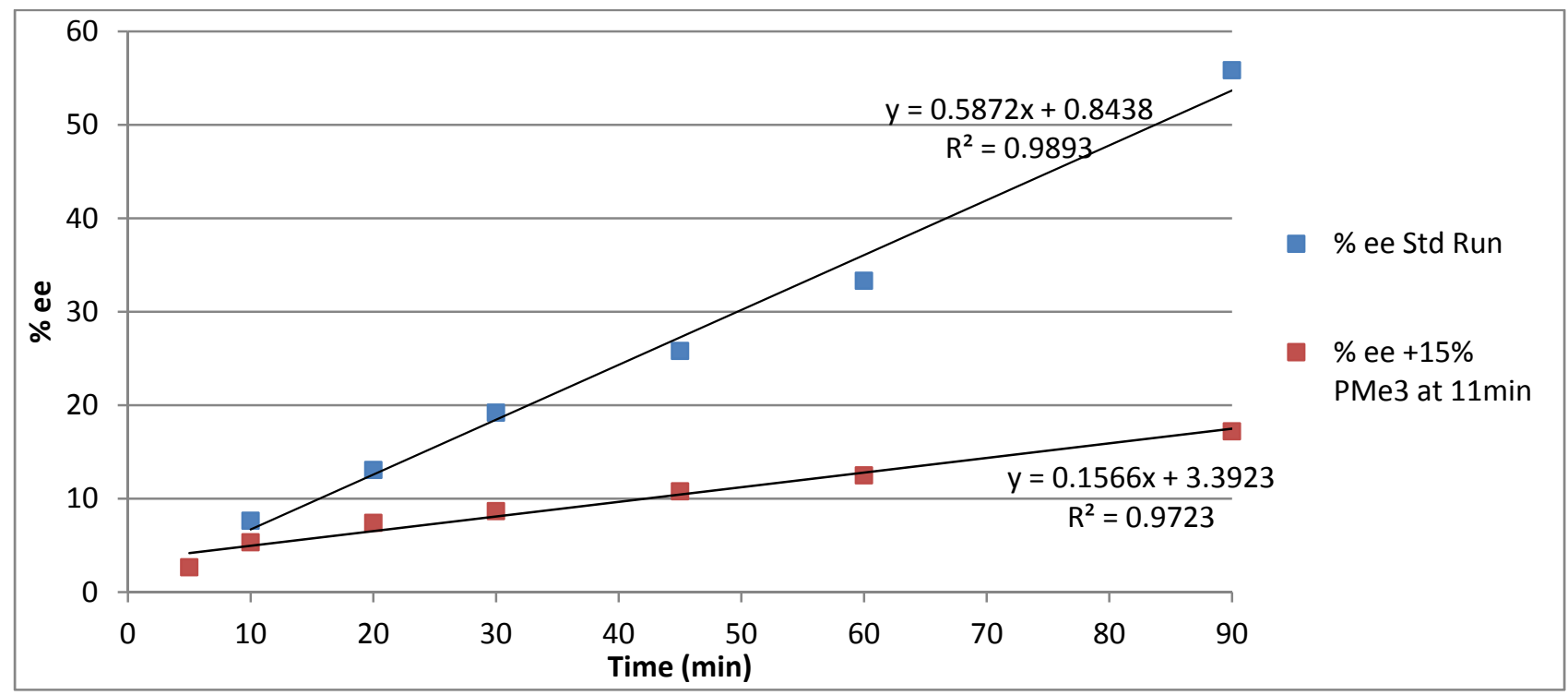

Figure 24: Reaction profiles for the catalytic oxidation of racemic 1-phenylethanol $(0.27 \mathrm{~mL}, 2.2 \mathrm{mmol})$ to acetophenone at room temperature $\left(28^{\circ} \mathrm{C}\right)$ in acetone $(6 \mathrm{~mL})$ using NaOiPr $(4 \mathrm{mg}, 0.049 \mathrm{mmol})$ run over one hour, and the poisoned runs using $\mathrm{PMe}_{3}(0.85 \mu \mathrm{mol}$ in $0.15 \mathrm{~mL} / 1.4 \mathrm{mmol})$ run over 5 hours.

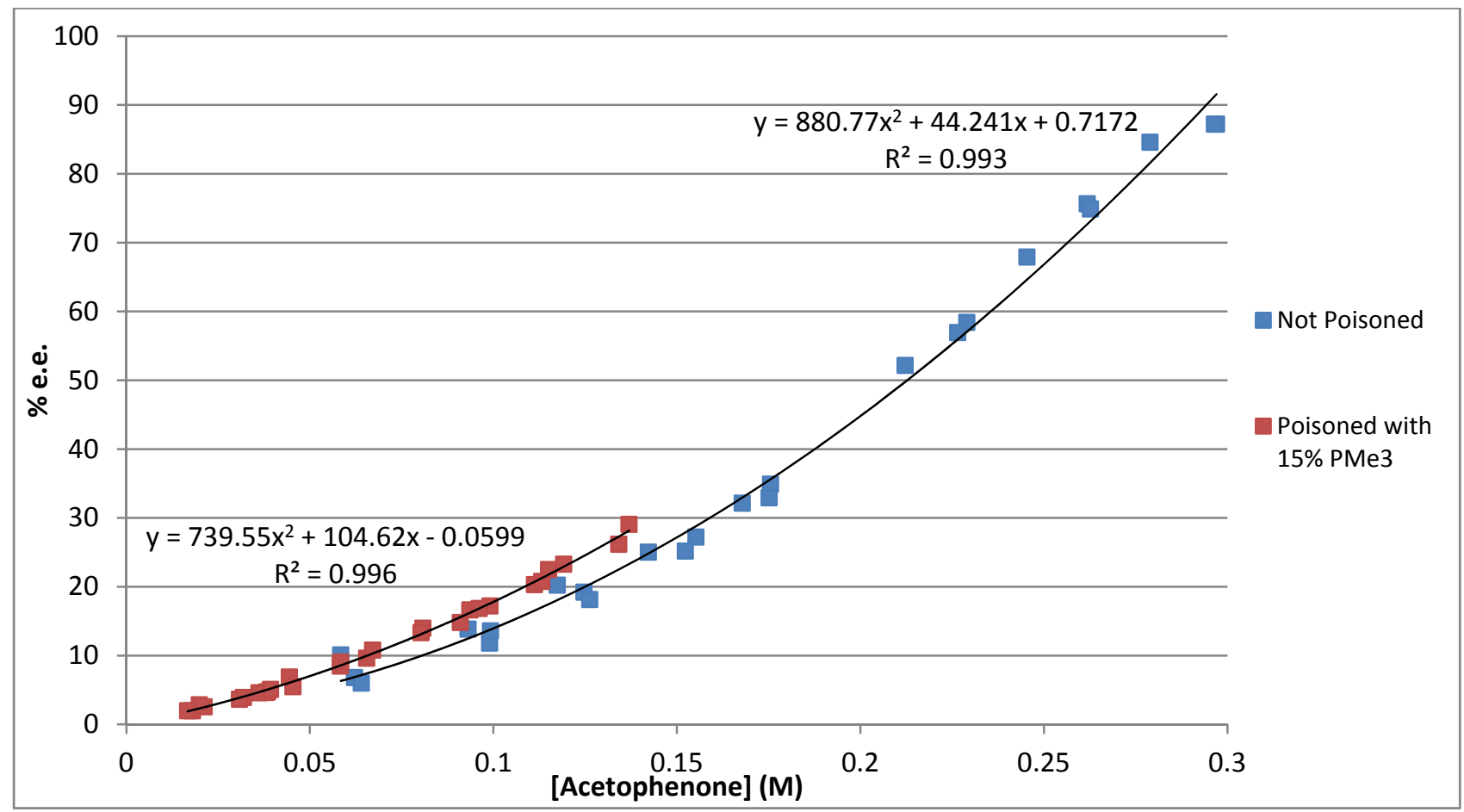


Figure 25: Reaction profiles for the catalytic oxidation of racemic 1-phenylethanol $(0.27 \mathrm{~mL}, 2.2 \mathrm{mmol})$ to acetophenone at $45^{\circ} \mathrm{C}$ in THF $(4.7 \mathrm{~mL})$ with benzophenone $(1.4 \mathrm{~g}, 7.7 \mathrm{mmol})$, and the poisoned runs using $\mathrm{PMe}_{3}(0.85 \mu \mathrm{mol}$ in $0.15 \mathrm{~mL} / 1.4$ mmol toluene).

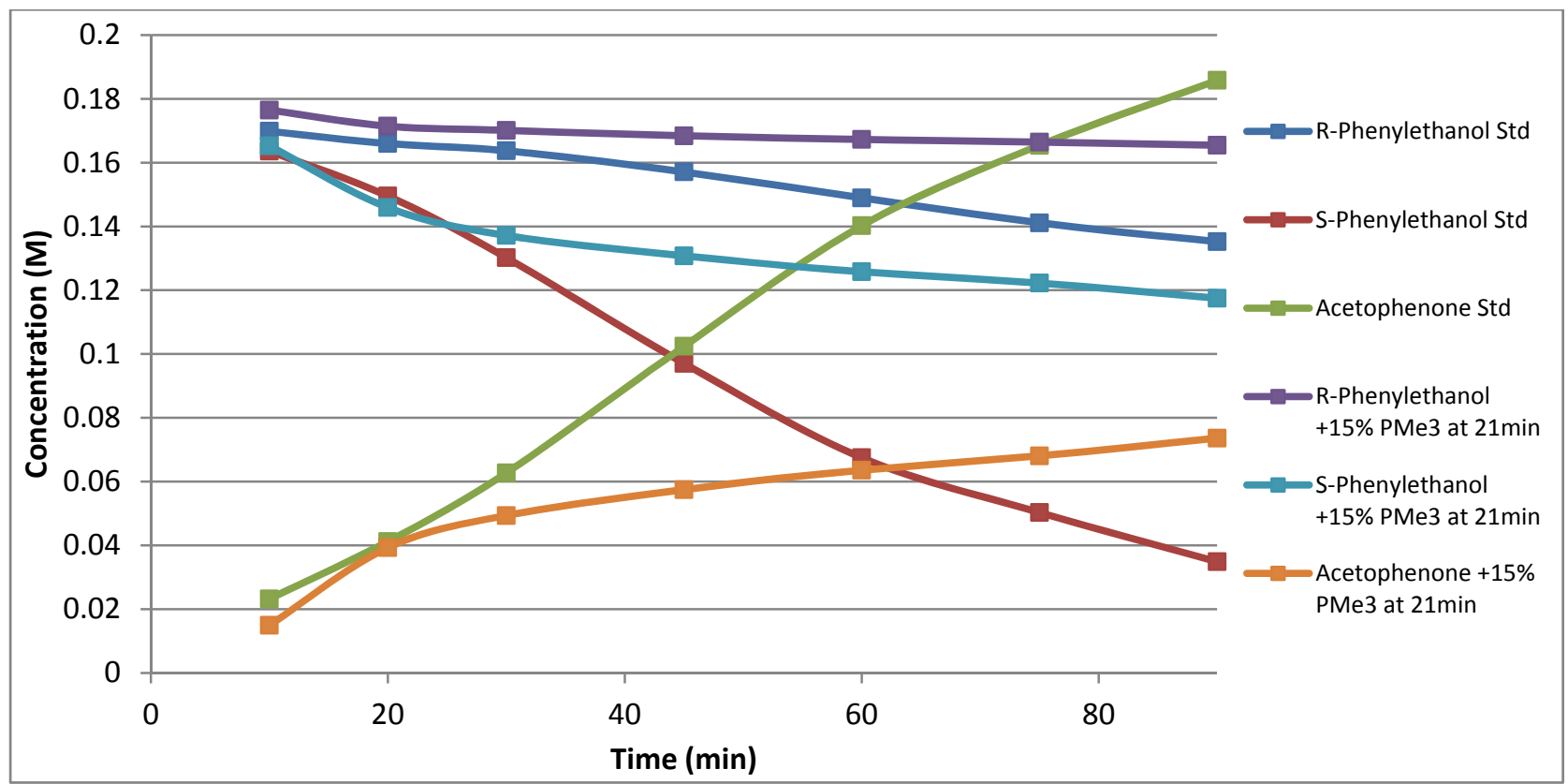

Figure 26: Enantiopurity profile for catalysis depicted in Figure 25.

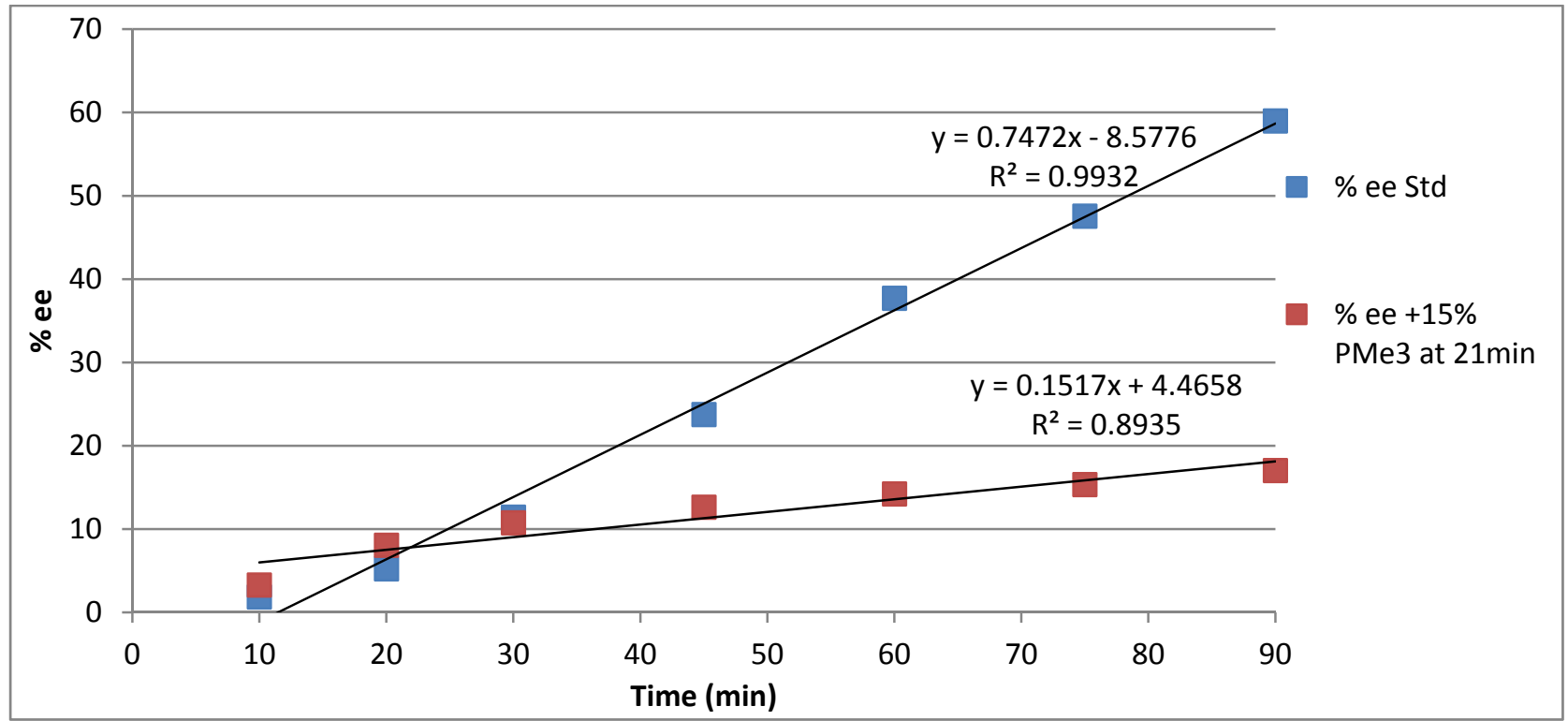


Figure 27: Reaction profiles for the catalytic oxidation of racemic 1-phenylethanol $(0.27 \mathrm{~mL}, 2.2 \mathrm{mmol})$ to acetophenone at $45^{\circ} \mathrm{C}$ in THF $(4.7 \mathrm{~mL})$ with benzophenone $(1.4 \mathrm{~g}, 7.7 \mathrm{mmol})$ run over one hour, and the poisoned runs using $\mathrm{PMe}_{3}(0.85 \mu \mathrm{mol}$ in $0.15 \mathrm{~mL} / 1.4 \mathrm{mmol}$ ) run over 5 hours.

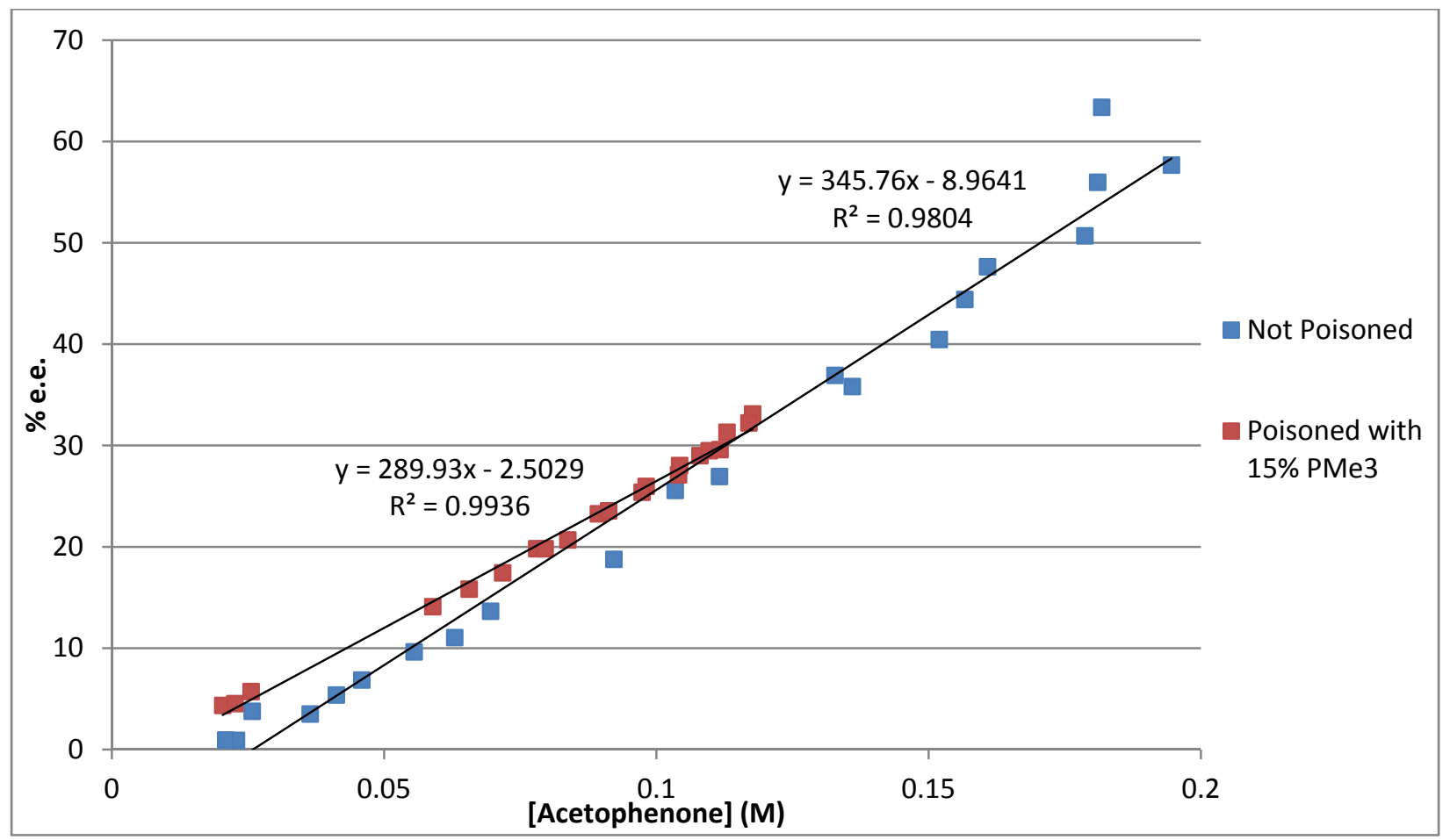




\section{Initiation Period Experiments}

Figure 28: Reaction profiles for the catalytic oxidation of racemic 1-phenylethanol $(0.27 \mathrm{~mL}, 2.2 \mathrm{mmol})$ to acetophenone at $45^{\circ} \mathrm{C}$ in THF $(4.7 \mathrm{~mL})$ with benzophenone $(1.4 \mathrm{~g}, 7.7 \mathrm{mmol})$, and runs in which the solvent or substrate were added at $45^{\circ} \mathrm{C}$ 10 minutes prior to reaction commencement.

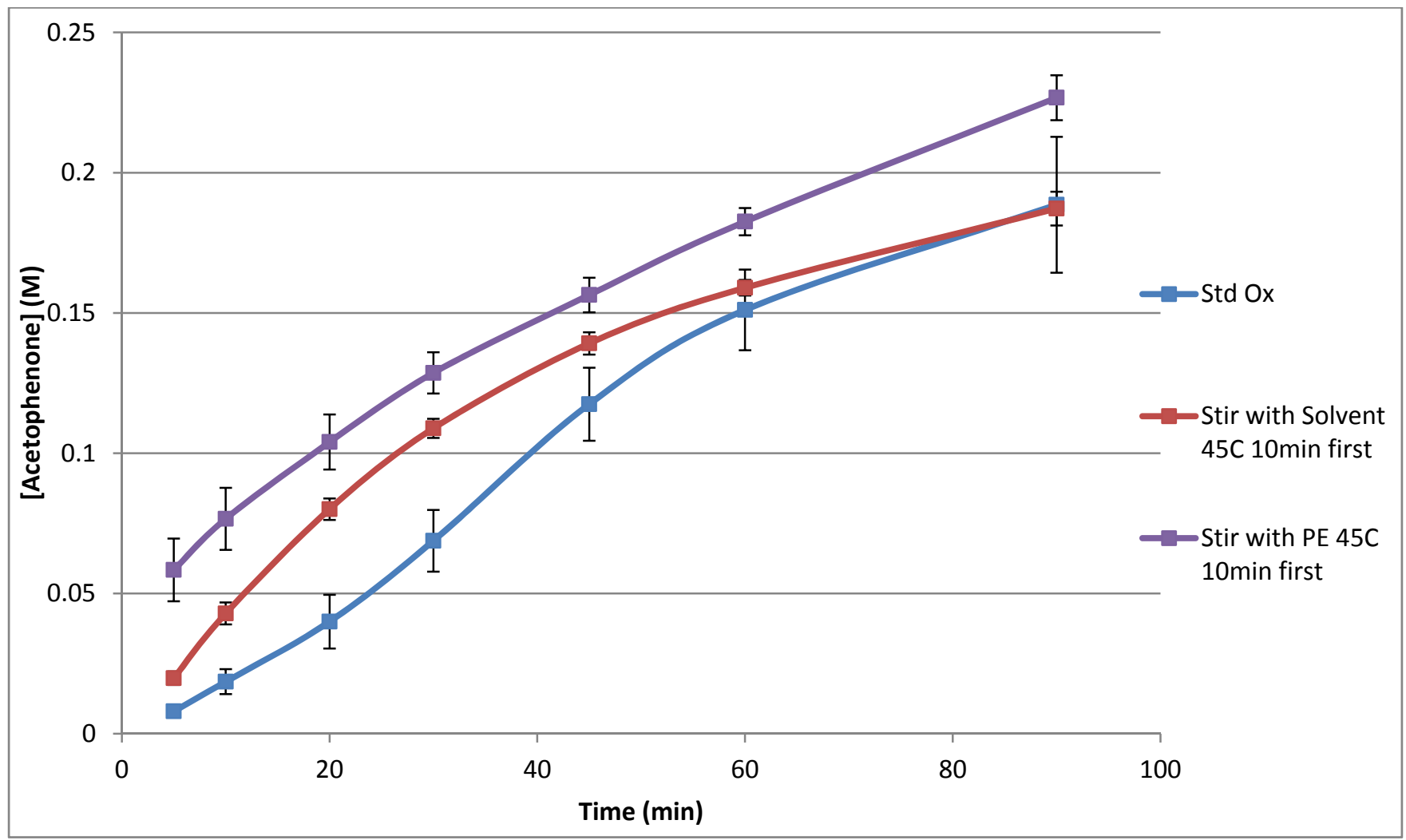

Figure 29: Enantiopurity profile for catalysis depicted in Figure 28.

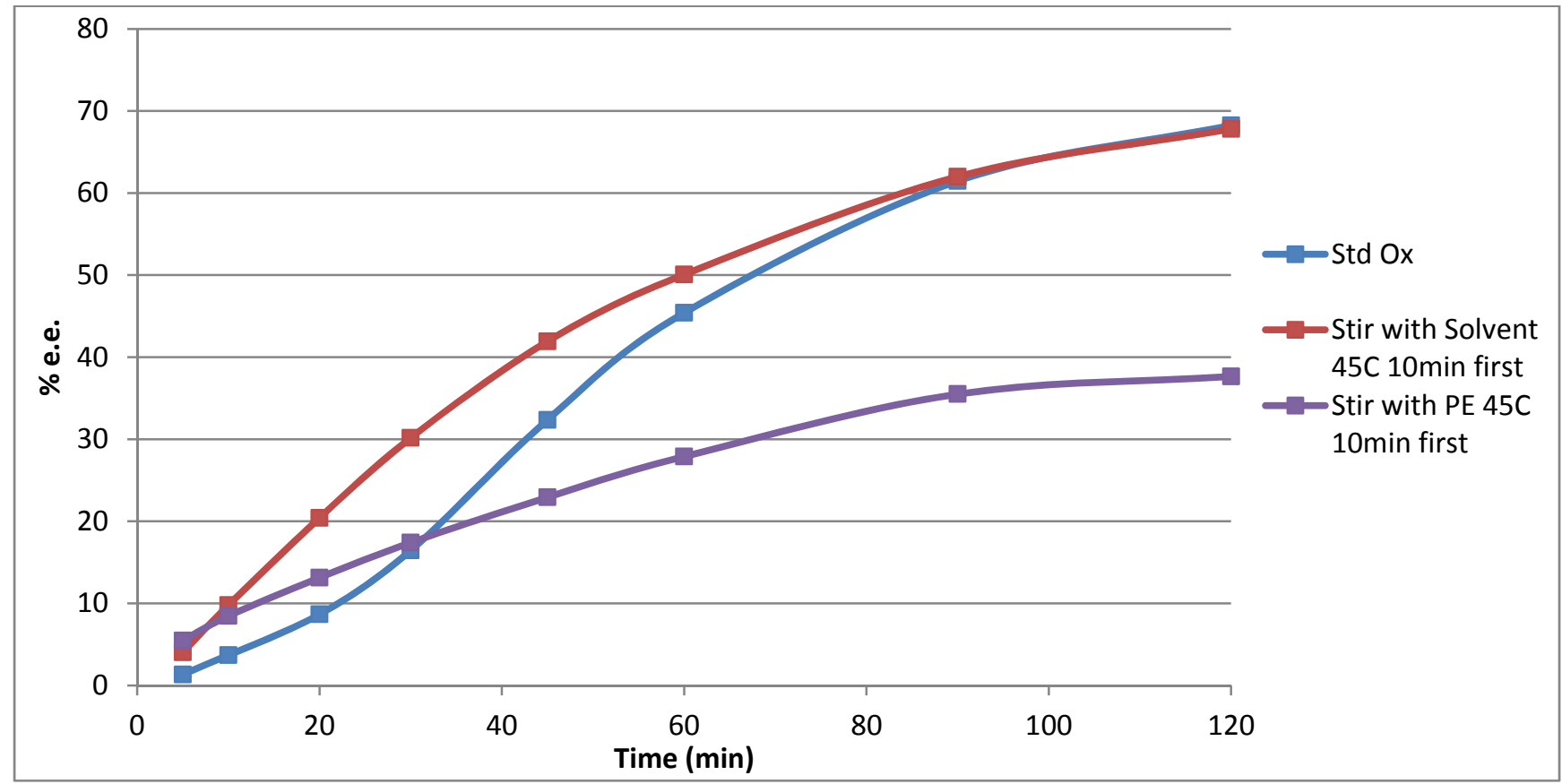




\section{Electron Microscopy Imaging}

Figure $30 \mathrm{a}$, b: STEM images taken at $-100^{\circ} \mathrm{C}$ of activated catalyst; solution prepared using (1) and NaOiPr at room temperature $\left(28^{\circ} \mathrm{C}\right)$ in acetone, using $\mathrm{iPrOH}$ as the substrate $(\mathrm{C}: \mathrm{B}: \mathrm{S}=1: 8: 230)$.
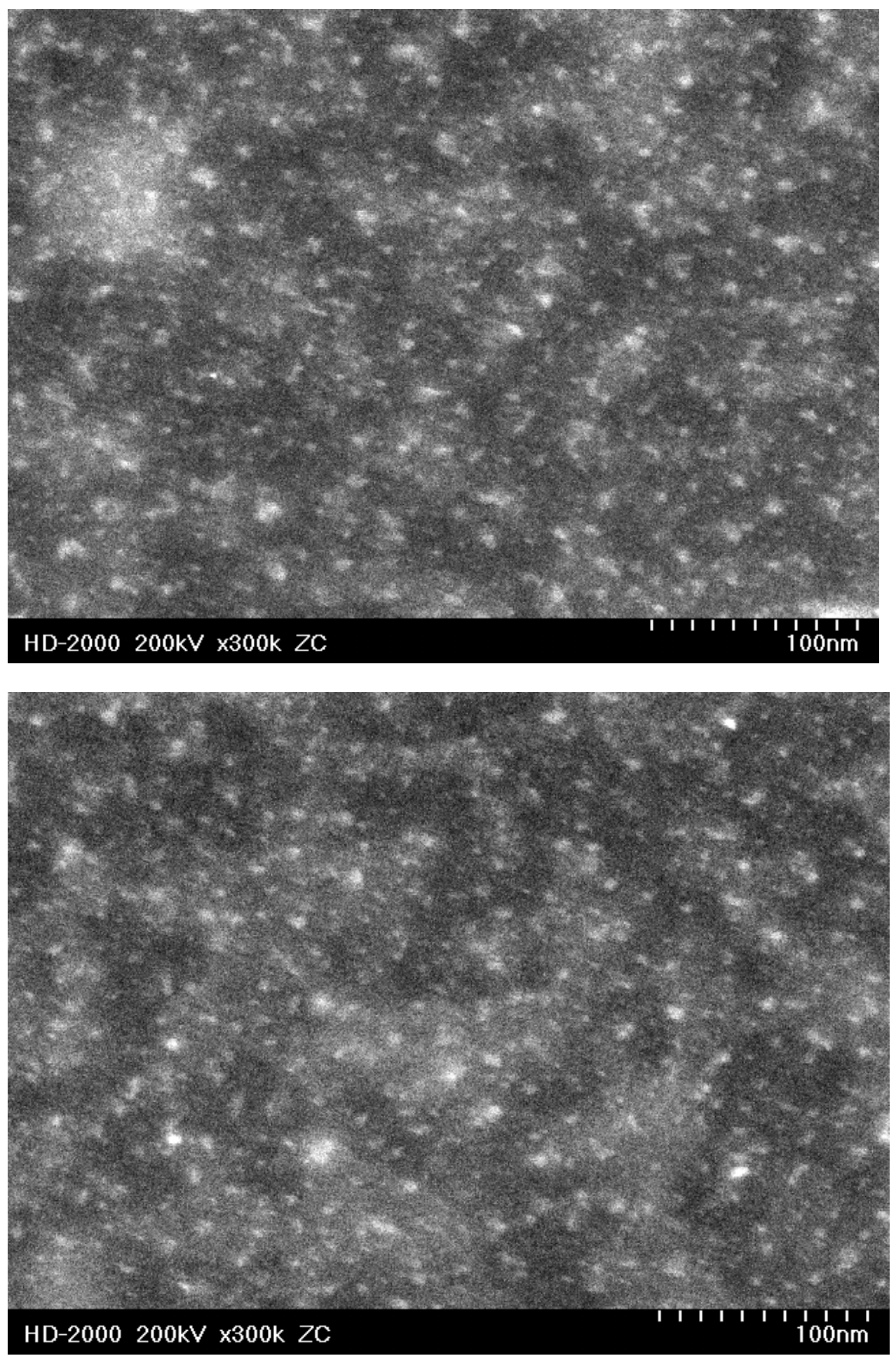
Figure $31 \mathrm{a}$, b: STEM images taken at $-100^{\circ} \mathrm{C}$ of activated catalyst; solution prepared using (1) and KOtBu at room temperature $\left(28^{\circ} \mathrm{C}\right)$ in THF/benzophenone, using iPrOH as the substrate (C:B:S = 1:8:230).
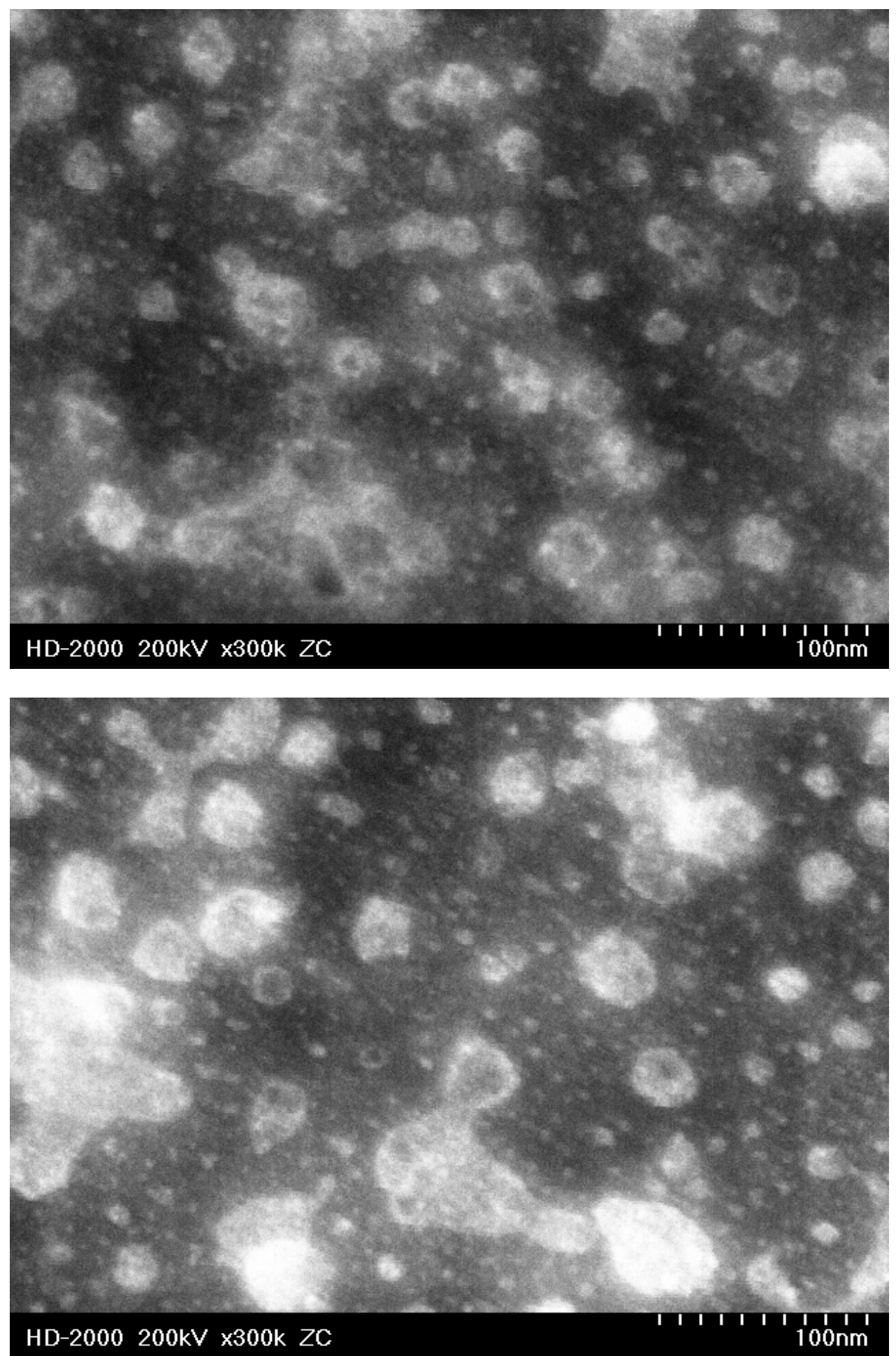


\section{Polymer-Supported Substrate Experiments}

\section{Polymer Functionalization and Catalysis}

Synthesis of Polymer-supported Aldehyde (Wang-Benzaldehyde, Wang-B)

StratoSpheres ${ }^{\mathrm{Tm}} \mathrm{PL}-$ Wang resin (1.0-1.5 mmol $-\mathrm{OH} / \mathrm{g}, 50-100$ mesh, $1 \%$ crosslinked with divinylbenzene) $(1.3 \mathrm{~g}, 1.95 \mathrm{mmol}-\mathrm{OH})$, was stirred with Pyridinium chlorochromate (PCC) $(420 \mathrm{mg}, 1.95 \mathrm{mmol})$ in $20 \mathrm{~mL}$ dichloromethane for 6 hours at room temperature in a nitrogen filled glovebox. The resin was then filtered under air, washed extensively with dichloromethane and isopropanol, and dried overnight under reduced pressure.

\section{Synthesis of Polymer-supported Alcohol (Wang-Phenylethanol, Wang-PE)}

Wang-B ( $600 \mathrm{mg}, 0.90 \mathrm{mmol}$-aldehyde) was swelled in $10 \mathrm{~mL}$ of dry tetrahydrofuran (THF) for 2 hours at room temperature under argon. A 3.0 M stock solution of methylmagnesiumchloride ( $\mathrm{MeMgCl}$ ) in ether $(0.48 \mathrm{~mL}, 1.44 \mathrm{mmol})$ was added slowly at $0^{\circ} \mathrm{C}$ under a constant flow of argon. The mixture was stirred at room temperature under argon for 20 hours, and then $2 \mathrm{~mL}$ of distilled water and $2 \mathrm{~mL}$ of isopropanol were added and stirred for another 30 minutes under air. The resin was then filtered under air, washed extensively with THF, water and isopropanol, and dried overnight under reduced pressure.

\section{Catalysis on Resin with (1)}

To a vial containing pre-catalyst (1) (12 mg, $0.0114 \mathrm{mmol}), \mathrm{NaOiPr}(8 \mathrm{mg}, 0.097 \mathrm{mmol})$, and Wang-PE ( $260 \mathrm{mg}, \sim 0.39 \mathrm{mmol}-\mathrm{OH})$, acetone $(4 \mathrm{~mL}, 54 \mathrm{mmol})$ and tetrahydrofuran $(2 \mathrm{~mL}, 25 \mathrm{mmol}$ ) were added at room temperature, in an argon filled glovebox. The solution was stirred for 24 hours, and then the resin was filtered under air, washed with isopropanol, THF and dichloromethane, and dried overnight under reduced pressure.

\section{Catalysis on Resin with PCC}

To a vial containing PCC (70 mg, $0.32 \mathrm{mmol}$ ) and Wang-PE ( $220 \mathrm{mg}, \sim 0.33 \mathrm{mmol}-\mathrm{OH}$ ), dichloromethane ( $6 \mathrm{~mL}, 94 \mathrm{mmol}$ ) was added at room temperature, in an argon filled glovebox. The solution was stirred for 16 hours, and then the resin was filtered under air, washed with isopropanol and dichloromethane, and dried overnight under reduced pressure. 


\section{NMR Spectra}

Figure 32: ${ }^{13} \mathrm{C}\left\{{ }^{1} \mathrm{H}\right\}$ solid state NMR of Wang Resin swollen in $\mathrm{CD}_{2} \mathrm{Cl}_{2}$, spun at $10 \mathrm{kHz}$

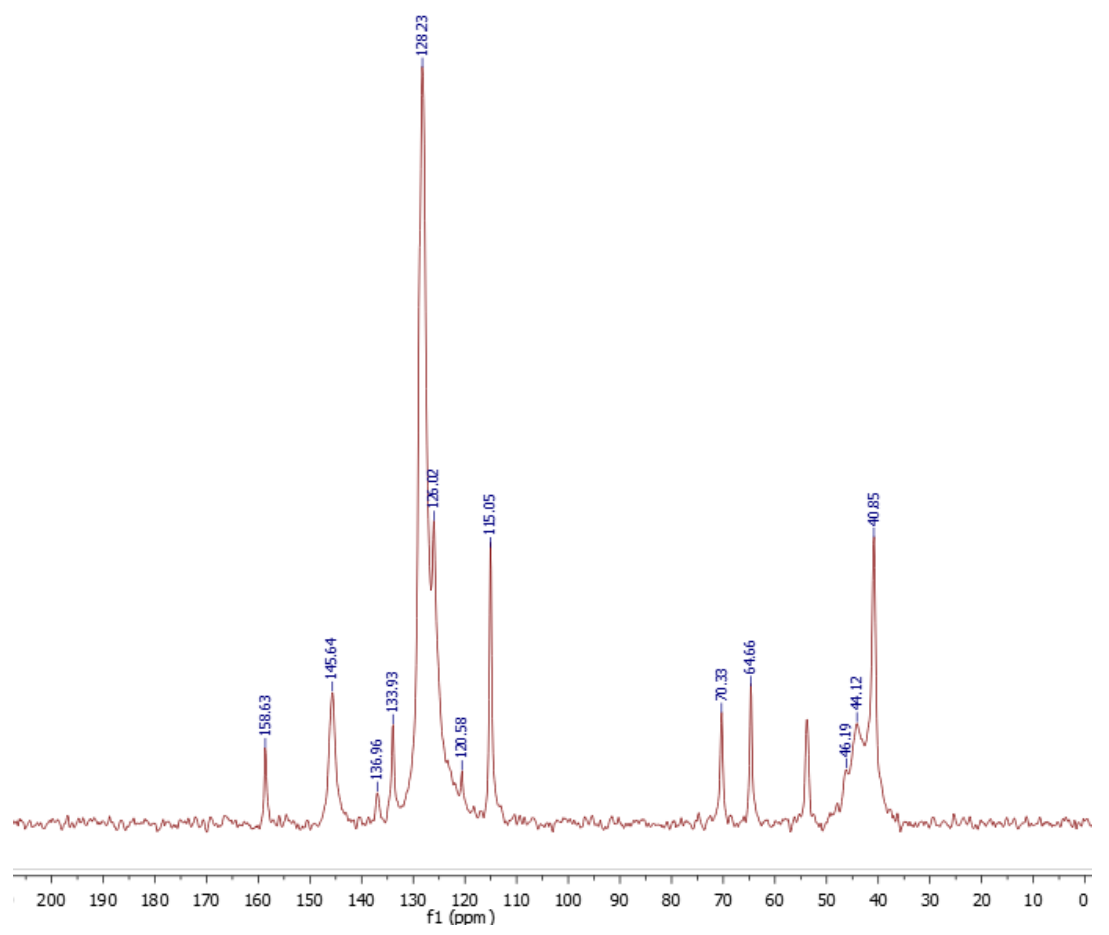

Figure 33: ${ }^{13} \mathrm{C}\left\{{ }^{1} \mathrm{H}\right\}$ solid state NMR of Wang-B swollen in $\mathrm{CD}_{2} \mathrm{Cl}_{2}$, spun at $10 \mathrm{kHz}$

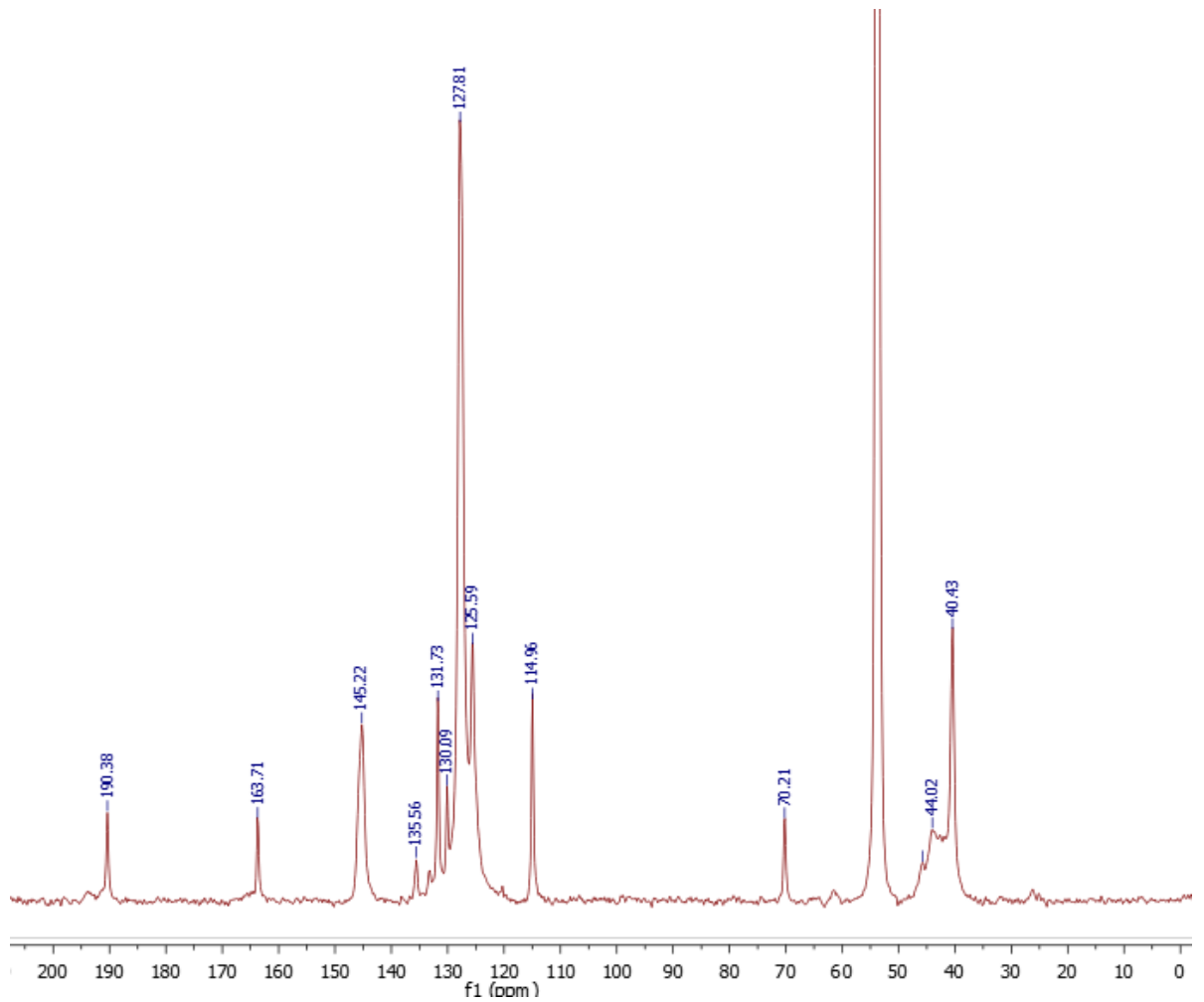


Figure $34:{ }^{13} \mathrm{C}\left\{{ }^{1} \mathrm{H}\right\}$ solid state NMR of Wang-PE swollen in $\mathrm{CD}_{2} \mathrm{Cl}_{2}$, spun at $10 \mathrm{kHz}$

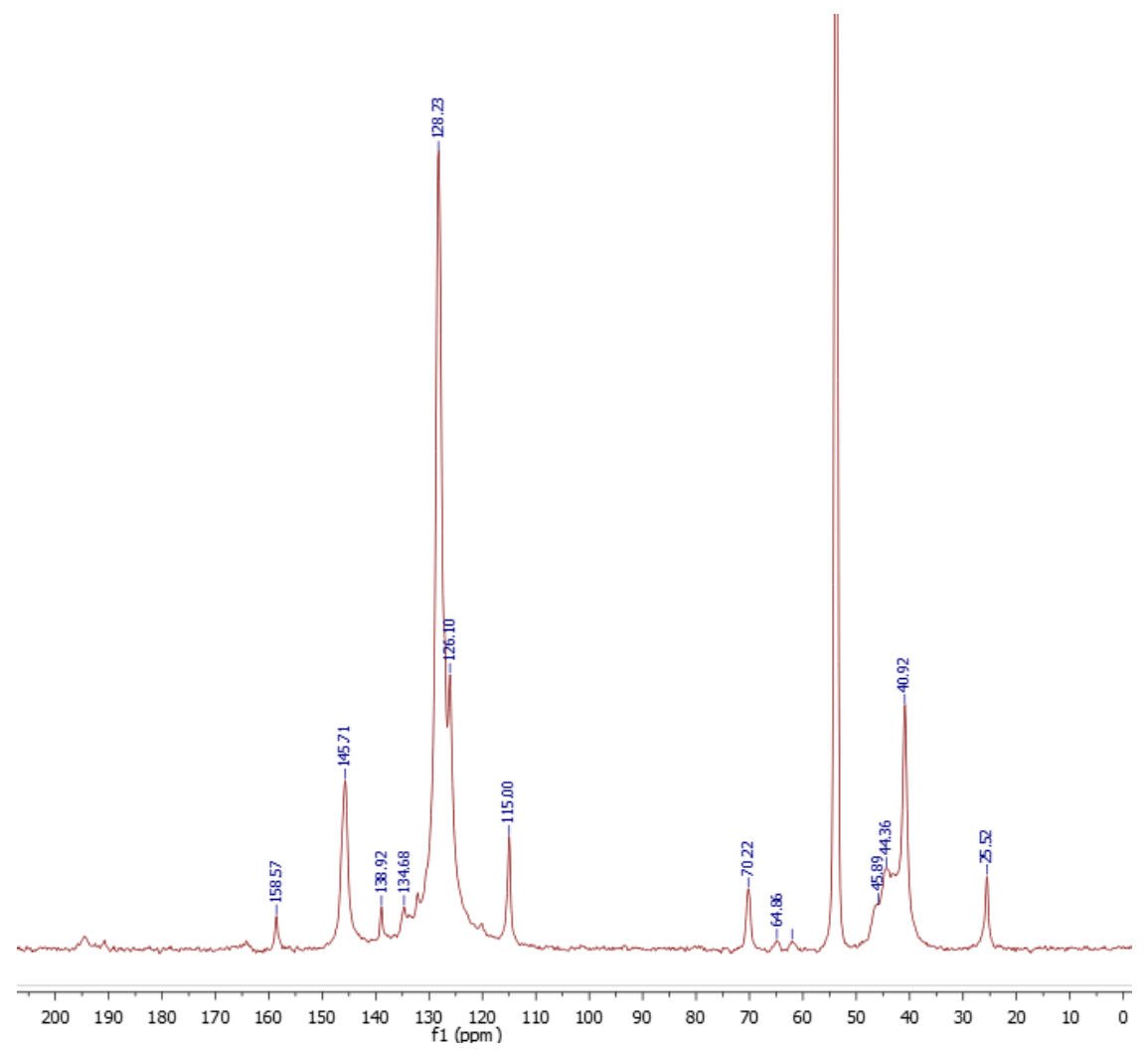

Figure 35: ${ }^{13} \mathrm{C}\left\{{ }^{1} \mathrm{H}\right\}$ solid state NMR of Wang-PE-Ox with (1), swollen in $\mathrm{CD}_{2} \mathrm{Cl}_{2}$, spun at $11 \mathrm{kHz}$

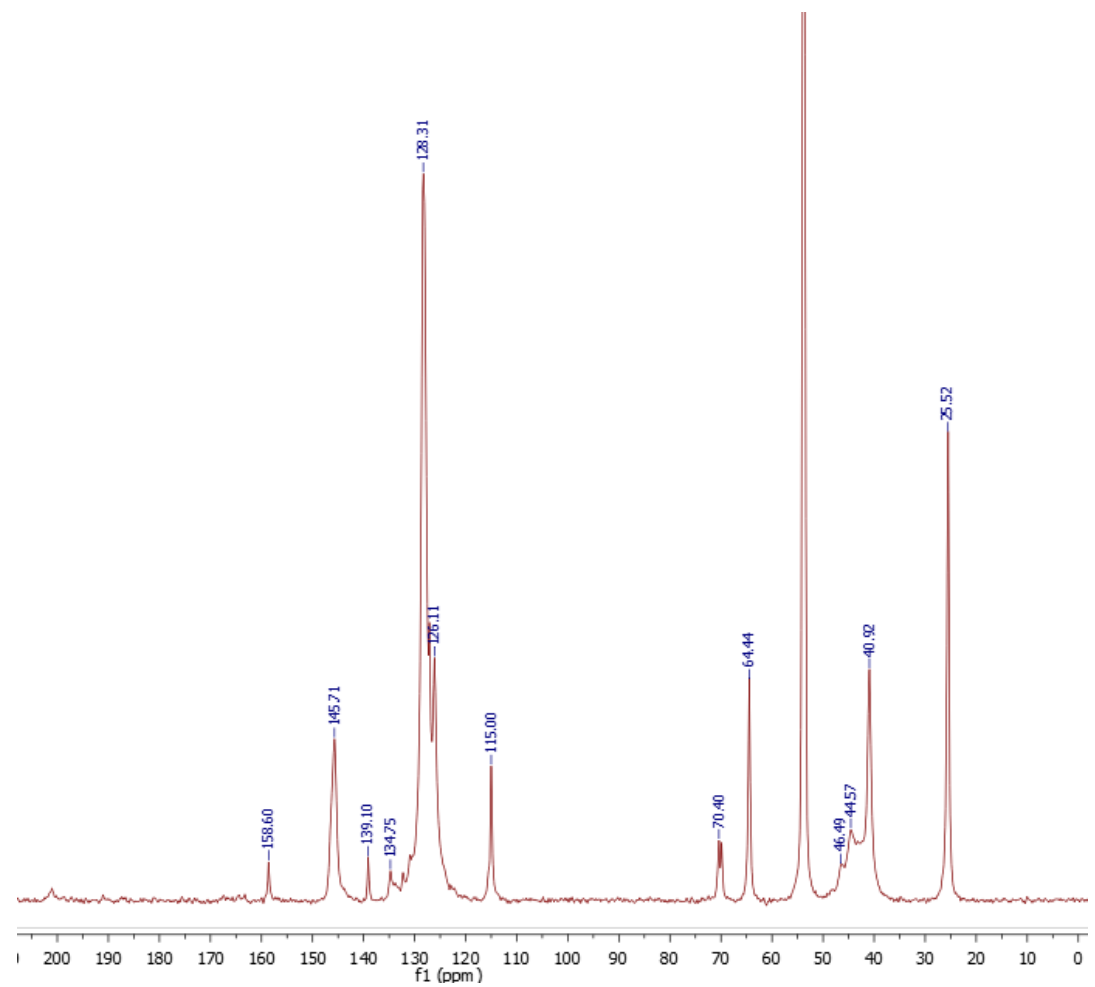


Figure 36: ${ }^{13} \mathrm{C}\left\{{ }^{1} \mathrm{H}\right\}$ solid state NMR of Wang-PE-Ox with PCC, swollen in $\mathrm{CD}_{2} \mathrm{Cl}_{2}$, spun at $12 \mathrm{kHz}$

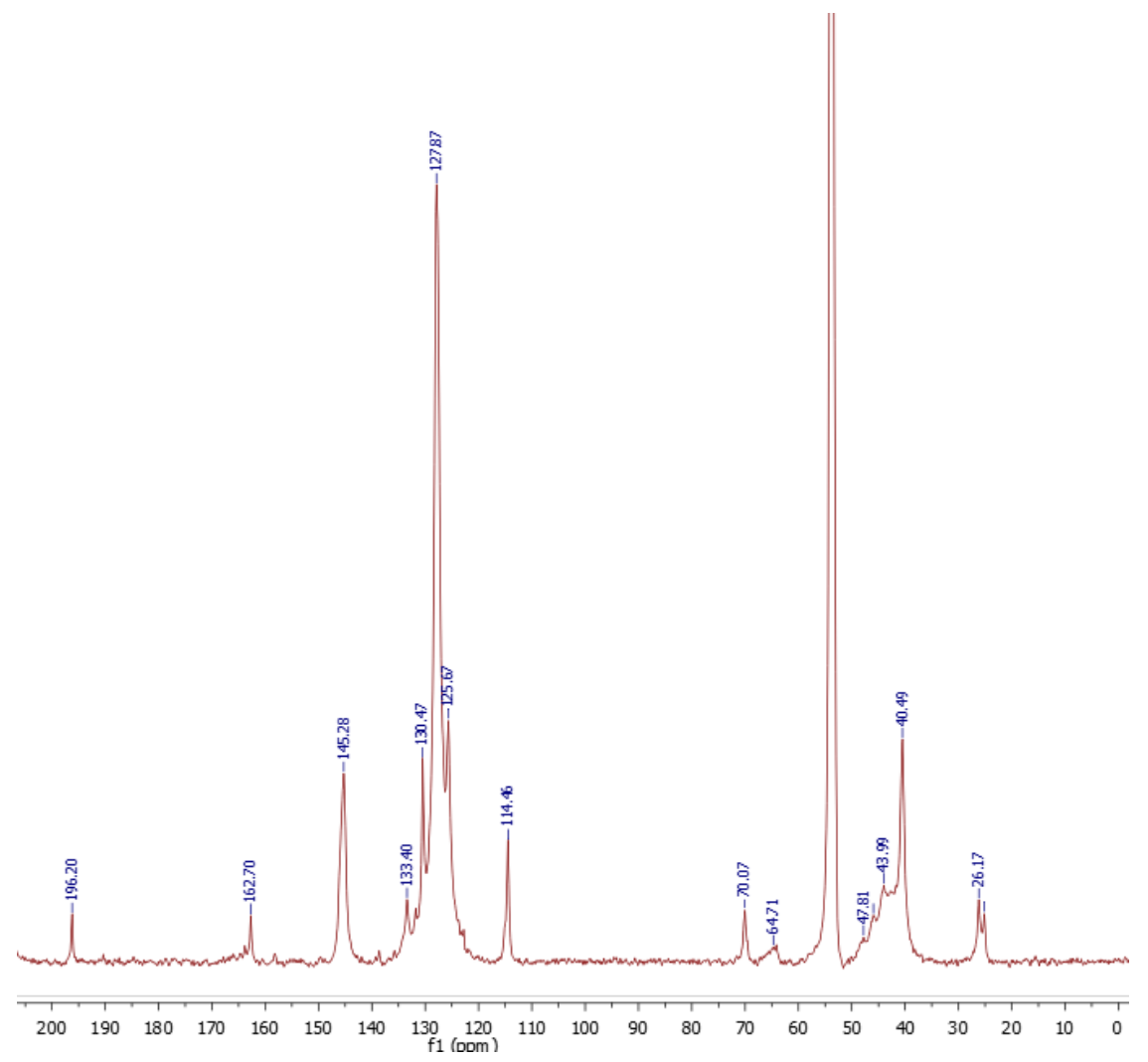

\section{References}

(1) Meyer, N.; Lough, A. J.; Morris, R. H. Chem. Eur. J. 2009, 15, 5605-5610.

(2) Sonnenberg, J. F.; Coombs, N.; Dube, P. A.; Morris, R. H. J. Am. Chem. Soc. 2012, 134, 5893-5899. 